\title{
Aggregation in age and space structured population models: an asymptotic analysis approach
}

\author{
J. BANASIAK, A. Goswami AND S. SHINDIN
}

Abstract. In this paper we describe how techniques of asymptotic analysis can be used in a systematic way to perform 'aggregation' of variables, based on a separation of different time scales, in a population model with age and space structure. The main result of the paper is proving the convergence of the formal asymptotic expansion to the solution of the original equation. This result improves and clarifies earlier results of Arino et al. (SIAM J Appl Math 60(2):408-436, 1999), Auger et al. (Structured population models in biology and epidemiology. Springer Verlag, Berlin, 2008), Lisi and Totaro (Math Biosci 196(2):153-186, 2005).

\section{Introduction}

Real systems can be modelled at various levels of resolution. For instance, a population can be described by giving the state of each individual and interactions between them (which we call the individual or microscopic level), by providing a statistic description of a sample of the system (which we shall call the mesoscopic, or kinetic, level), and also by averaging over mesoscopic (structural) states; that is, at the level of interactions between subpopulations of the original system (which we term the macroscopic or hydrodynamic level).

It is clear that the microscopic description provides the most detailed information but at a considerable, if not insurmountable, computational cost. Also, in many cases such a detailed information is redundant. On the other hand, the macroscopic description typically involves measurable quantities, so that the analysis and computations immediately can be verified by experiment, and it is computationally less involved. However, for some applications, it may be too crude. Thus, in recent years, with computational power easily available, the mesoscopic (or kinetic) descriptions have become increasingly popular.

In practice, when given a detailed microscopic system with various interacting organizational levels, we are faced with the question of how to collect the variables to create

Mathematics Subject Classification (2000): 92D25, 35B25, 35Q80, 47D06, 47N20

Keywords: Structured population models, Aggregation, Singular perturbation, Asymptotic analysis, Semigroups.

The work of all authors was supported by the National Research Foundation of South Africa under grant FA2007030300001 and the University of KwaZulu-Natal Research Fund. 
an aggregated system on a lower level of complexity which, nevertheless, preserves relevant features of the original one. Unfortunately, due to intertwining of variables, in most cases the aggregation of variables leads to models which are qualitatively different from the microscopic one and the aggregated dynamics can provide only an approximation of the original one. Problems of this type are called singularly perturbed and various techniques have been developed to facilitate passages between different regimes. In this paper we shall focus on a broadly understood asymptotic analysis which, though one of many, in our opinion is the easiest and the most systematic method on both conceptual and implementation levels.

Our main interest are models coming from structured population biology, where we can observe various levels of organization such as individual, population, community or ecosystem. What makes the aggregation of variables from one level to another possible is the existence of different time scales at which each level evolves. For example, individual time scale is usually much faster than the demographic one. Thus the ratio of time scales can be used as the parameter separating the regimes in which the system operates. This is the approach we adopt in this paper, where we analyze a McKendrick type system of equations describing an age-structured population which is additionally subdivided into several groups. These groups could refer to a geographical location, as in the original model describing evolution and migration of sole, [2], but could have other meaning: one can consider a population of cells subdivided according to the number of genes of a particular type they have. A similar structure is displayed by epidemiological models with age structure, [12, p. 113]. Migration between the patches is assumed to occur at a much faster time scale than the demographic processes such as aging; this is reflected in the model by introducing a large parameter $1 / \epsilon$ in front of the transition matrix. The resulting equation is, [2,3,9],

$$
\mathbf{n}_{t}:=\mathcal{S} \mathbf{n}-\mathcal{M} \mathbf{n}+\frac{1}{\epsilon} \mathcal{C} \mathbf{n},
$$

where subscript $t$ denotes differentiation with respect to $t$,

$$
\mathbf{n}(t, a)=\left(n_{1}(t, a), \ldots, n_{N}(t, a)\right)
$$

and $n_{i}(t, a)$ is the population density at time $t$ of individuals residing in patch $i$ and being of age $a$. Further, $\mathcal{S} \mathbf{n}=-\mathbf{n}_{a}$ describes aging, $\mathcal{M}(a)=\left\{\mu_{i}(a)\right\}_{1 \leq i \leq N}$ is the mortality matrix and the matrix $\mathcal{C}=\left\{c_{i j}(a)\right\}_{1 \leq i, j \leq N}$ describes the transfer of individuals between patches.

This system is supplemented by the McKendrick boundary condition

$$
[\gamma \mathbf{n}](t)=\mathbf{n}(t, 0)=[\mathcal{B} \mathbf{n}](t)=\int_{0}^{\infty} B(a) \mathbf{n}(t, a) d a,
$$

where $\gamma$ denotes the operator of taking the trace at $a=0$ and $B(a)=\left\{\beta_{i j}(a)\right\}_{1 \leq i, j \leq N}$ is the fertility matrix. The initial condition is given by

$$
\left.\mathbf{n}\right|_{t=0}=\mathbf{n}(0, a)=\stackrel{\circ}{\mathbf{n}}(a) .
$$


We consider (1.1)-(1.3) in the space $\mathbf{X}=L_{1}\left(\mathbb{R}_{+}, \mathbb{R}^{N}\right)$, where the norm of a non-negative element gives the total population. To avoid multiplying notation, we shall use the same notation, say $\mathbf{n}$, to denote the $\mathbf{X}$-valued function $t \rightarrow \mathbf{n}(t)$ as well as the $\mathbb{R}^{N}$-valued function $(t, a) \rightarrow \mathbf{n}(t, a)$. We note that typically in linear models the matrix $\mathcal{M}$ is diagonal: $\mathcal{M}(a)=\operatorname{diag}\left\{\mu_{1}(a), \ldots, \mu_{N}(a)\right\}$ which reflects the fact that death is an intra-patch phenomenon (in nonlinear models death coefficients often depend on the total population). However, linear models with general matrix $\mathcal{M}$ are also considered, see [19, Assumption (4.68)], and since it will not affect our results, the analysis covers such matrices. On the other hand, births in a particular patch can easily depend on the population density in other patches (e.g. females could move to a safer patch just to give birth) and thus considering full matrix $B$ is perfectly reasonable. This makes our analysis more general than that in $[2,15]$, where only diagonal matrices $\mathcal{M}$ and $B$ are considered.

Biological heuristics suggests that no geographical structure should persist for very large interstate transition rates; that is, for $\epsilon \rightarrow 0$. Precise assumptions on the problem are provided in Sect. 2, here we only note that both biological and mathematical analyses rely on $\lambda=0$ being the dominant simple eigenvalue of $\mathcal{C}(a)$ for each $a \in \mathbb{R}_{+}$with a corresponding positive right eigenvector, denoted by $\mathbf{k}(a)$, and the left eigenvector $\mathbf{1}=(1,1, \ldots, 1), \mathbf{k}(a)$ is normalized to satisfy $\mathbf{1} \cdot \mathbf{k}=1$. The vector $\mathbf{k}(a)=\left(k_{1}(a), \ldots, k_{N}(a)\right)$ is the so-called stable patch structure; that is, the asymptotic (as $t \rightarrow \infty$ and disregarding demographic processes) distribution of the population among the patches for a given age $a$. Thus, in population theory the components of $\mathbf{k}$ are approximated by $k_{i} \approx n_{i} / n$ for $i=1, \ldots, N$, where

$$
n=\mathbf{n} \cdot \mathbf{1}=\sum_{n=1}^{N} n_{i} \text {. }
$$

Adding together equations in (1.1) and using the above we obtain

$$
n_{t} \approx-n_{a}-\mu^{*} n
$$

where $\mu^{*}=\mathbf{1} \cdot \mathcal{M} \mathbf{k}$ is the 'aggregated' mortality. This model, supplemented with the boundary condition

$$
n(t, 0) \approx \int_{0}^{\infty} \beta^{*}(a) n(t, a) d a,
$$

where $\beta^{*}=\mathbf{1} \cdot B \mathbf{k}$, is called the aggregated model, and is expected to provide an approximate description of the averaged population. Thus, (1.5) is the macroscopic and (1.1) the mesoscopic description of the population.

The main result of the paper is a rigorous validation of the above heuristics; that is, that the true total population $n$ can be approximated by the solution $\bar{n}$ of the aggregated problem (1.5)-(1.6) (where ' $\approx$ ' is replaced by ' $=$ ') with an $\epsilon$-order error. The analysis is involved due to the initial and boundary conditions which are not consistent with those of the aggregated model. This makes the problem singularly perturbed and thus 
necessitates a careful analysis of the boundary, corner and initial layer phenomena. We are able to prove that if the solution $\mathbf{n}$ to (1.1) is decomposed according to

$$
\mathbf{n}=n \mathbf{k}+\mathbf{w}
$$

where $n$ is defined by (1.4), with analogous decomposition of the initial condition $\stackrel{\circ}{\mathbf{n}}=\stackrel{\circ}{n} \mathbf{k}+\stackrel{\circ}{\mathbf{w}}$, and $\bar{n}$ is the solution of the scalar aggregated problem, then for any (sufficiently small $\epsilon$ ) and any time interval $[0, T]$, there is a constant $C(T, \mathcal{M}, B, \mathcal{C})$ such that

$$
\begin{gathered}
\|n(t, \cdot)-\bar{n}(t, \cdot)\|_{L_{1}\left(\mathbb{R}_{+}\right)} \leq \epsilon C(T, \mathcal{M}, B, \mathcal{C})\|\stackrel{\circ}{\mathbf{n}}\|_{W_{1}^{1}\left(\mathbb{R}_{+}, \mathbb{R}^{N}\right)}, \\
\left\|\mathbf{w}(t, \cdot)-e^{\frac{t}{\epsilon} \mathcal{C}(\cdot)} \stackrel{\circ}{\mathbf{w}}(\cdot)\right\|_{L_{1}\left(\mathbb{R}_{+}, \mathbb{R}^{N}\right)} \leq \epsilon C(T, \mathcal{M}, B, \mathcal{C})\|\stackrel{\circ}{\mathbf{n}}\|_{W_{1}^{1}\left(\mathbb{R}_{+}, \mathbb{R}^{N}\right)},
\end{gathered}
$$

uniformly in $t \in[0, T]$. Here $W_{1}^{1}$ denotes the standard Sobolev space. We note that $e^{\frac{t}{\epsilon} \mathcal{C}(\cdot)}$ is of negative type since 0 is the dominant eigenvalue of $\mathcal{C}$. Thus this term provides the initial layer of the problem. Furthermore, using equiboundedness of the involved operators with respect to $\epsilon$ and density of $W_{1}^{1}$ in $L_{1}$ we can extend the convergence to arbitrary initial conditions losing, however, the rate of convergence. We note, that (1.7) and (1.8) show that the above problem is an example of a degenerate convergence problem the regular part of which can be considered within the framework of the Sova-Kurtz version of the Trotter-Kato theory, $[6,8]$.

Aggregation for (1.1) has been studied quite extensively in [2,3,9] and in [15]. The results of the former are similar to $(1.7)$ and $(1.8)$, see $(*)-(* * *)$ in [2, p. 427]. However, to get estimates valid up to $t=0$, the authors used the solution of the full problem restricted to the manifold complementary to $\mathbf{k}(a)$ so that in practice finding the approximation presents difficulties comparable to solving the original problem. In our approach the asymptotic analysis provides the necessary correction in a systematic way as an explicit solution of a linear autonomous system of ordinary differential equations so that using this approximation is computationally viable. Moreover, there are some gaps in the argument of [2], one of them being that the projected boundary conditions in [2] are correct only if $\mathbf{k}$ is independent of age (compare [2, Eq. (3.4)] with (3.4)). Moreover, classical solutions to (1.1) and (1.5) exist only with initial data satisfying nonlocal compatibility conditions and, unless additional necessary constraints are imposed on the initial data, both problems should be considered in their mild form, as discussed in Sect. 4. This approach, though computationally more involved, allows to remove several technical assumptions imposed in [2].

We note that the asymptotic expansion techniques were employed in [15] the authors, however, have not proved the convergence of the expansion; also the the layers which are left depending explicitly on $\epsilon$, are not completely correct.

The paper is organized as follows. In Sect. 2 we provide the assumptions and basic properties of the model. Section 3 contains construction of the formal asymptotic expansion and the formal error equation. The construction is carried out gradually with full explanation of each step and thus it can serve as a brief tutorial of the method. 
We note that the structure of the problem makes the asymptotic expansion similar to the Chapman-Enskog method which is well known in the kinetic theory, see e.g. [5,6,17], from where some terminology and notation were borrowed. We again emphasize that the classical differential equation formulation of the problem is insufficient due to discontinuities of solutions resulting from the nonlocal boundary conditions and thus in Sect. 4 we develop the integral formulation of the problem based on the construction in [19]. In Sect. 5 we prove that the formal asymptotic expansion converges to the solution of the aggregated problem and Sect. 6 contains a numerical illustration of the results.

\section{Preliminary properties of (1.1)}

Let us discuss problem (1.1)-(1.3) in more detail. We assume that $a \rightarrow B(a)$ is a measurable bounded matrix function on $\mathbb{R}_{+}$and $a \rightarrow \mathcal{M}(a) \in C_{b}^{1}\left(\mathbb{R}_{+}, \mathbb{R}^{N^{2}}\right)$ (differentiable functions with bounded derivatives). Furthermore, we assume that $-\mathcal{M}(a)$ is a sub-Kolmogorov matrix, that is, it is positive off-diagonal and satisfies $-\sum_{j=1}^{N} \mu_{j i}(a) \leq 0$ for any $1 \leq i \leq N$ and $a \in \mathbb{R}_{+}$. Thus, $-\mathcal{M}(a)$ generates a positive semigroup of contractions in $\mathbb{R}^{N}$ for each $a \in \mathbb{R}_{+}$and hence $-\mathcal{M}$ generates a positive semigroup of contractions the space $\mathbf{X}=L_{1}\left(\mathbb{R}_{+}, \mathbb{R}^{N}\right)$.

Further, we assume that $a \rightarrow \mathcal{C}(a) \in C_{b}^{2}\left(\mathbb{R}_{+}, \mathbb{R}^{N^{2}}\right)$ and for each $a \in \mathbb{R}_{+}$the matrix $\mathcal{C}(a)$ is the so-called ML-matrix, that is, it is positive off-diagonal, irreducible and satisfies $\sum_{j=1}^{N} c_{j i}(a)=0$ for any $1 \leq i \leq N$ and $a \in \mathbb{R}_{+}$, [18].

Before we move to asymptotic properties of (1.1) we need to recall basic facts on its solvability. We shall go deeper into the theory later when needed. It follows, [19, Proposition 3.2], that $\mathcal{S}-\mathcal{M}+\epsilon^{-1} \mathcal{C}$ on the domain $D(\mathcal{S})=\{\mathbf{u} \in \mathbf{X} ; \gamma \mathbf{u}=\mathcal{B} \mathbf{u}\}$ generates a semigroup, say $\left(\mathcal{G}_{\epsilon}(t)\right)_{t \geq 0}$, of type $(1, \omega)$ where $\omega \leq\|\mathcal{B}\|+\left\|-\mathcal{M}+\epsilon^{-1} \mathcal{C}\right\|$. This estimate is not satisfactory as it depends on $\epsilon$. However, $-\mathcal{M}+\epsilon^{-1} \mathcal{C}$ is also positive off-diagonal and hence it generates a positive semigroup of contractions. Thus the assumptions of the Trotter formula, [11, Corollary III 5.8]), are satisfied and therefore the type of $\left(\mathcal{G}_{\epsilon}(t)\right)_{t \geq 0}$ is the same as of the semigroup generated by $(\mathcal{S}, D(\mathcal{S}))$. Hence $\omega \leq\|\mathcal{B}\|$, independently of $\epsilon$.

\subsection{Spectral properties of $\mathcal{C}$}

The assumptions on $\mathcal{C}$ ensure that for each $a \in \mathbb{R}_{+}, 0$ is the simple dominant eigenvalue of $\mathcal{C}(a)$ with a positive eigenvector $\mathbf{k}(a)$. The null-space of the adjoint matrix is spanned by $\mathbf{1}=(1,1, \ldots, 1)$ and we will normalize $\mathbf{k}$ to satisfy

$$
\mathbf{1} \cdot \mathbf{k}=1 \text {. }
$$

In this case the ( $a$-dependent) spectral projection $\mathcal{P}$ onto $\mathbf{k}(a)$ is given by

$$
\mathcal{P} \mathbf{f}=(\mathbf{f} \cdot \mathbf{1}) \mathbf{k}=\mathbf{k} \sum_{n=1}^{N} f_{i},
$$


while the complementary projection is given by $\mathcal{Q} \mathbf{f}=\mathbf{f}-(\mathbf{f} \cdot \mathbf{1}) \mathbf{k}$. The 'eigenspace' corresponding to $\lambda=0$ is $a$-dependent and is given as $V=\operatorname{Span}\{\mathbf{k}\}$. However, the complementary space to $V$ is independent of $a$ and it is given by $W=\operatorname{Im} \mathcal{Q}=$ $\{\mathbf{x} ; \mathbf{1} \cdot \mathbf{x}=0\}$. Hence any element $\mathbf{n} \in \mathbb{R}^{N}$ can be decomposed as

$$
\mathbf{n}=\mathcal{P} \mathbf{n}+\mathcal{Q} \mathbf{n}=\mathbf{v}+\mathbf{w}=n \mathbf{k}+\mathbf{w},
$$

where $n$ is a scalar. For each $a \in \mathbb{R}_{+}$the decomposition $\mathbb{R}^{N}=V(a) \oplus W$ reduces $\mathcal{C}(a)$. The part in $V$ is zero whereas for $\mathcal{C}_{W}:=\mathcal{Q C} \mathcal{Q}=\left.\mathcal{C}\right|_{W}$ we have $s\left(\mathcal{C}_{W}(a)\right)=$ $\max \left\{\Re \lambda(a), \lambda(a) \in \sigma\left(\mathcal{C}_{W}(a)\right)\right\}<0$. For the asymptotic analysis of (1.1) we need

$$
\sup _{a \in \mathbb{R}_{+}} s\left(\mathcal{C}_{W}(a)\right)=: s_{C}<0 .
$$

LEMMA 2.1. Under the above assumptions, $\mathcal{C}_{W}^{-1} \in C_{b}^{2}\left(\mathbb{R}_{+}, \mathbb{R}^{N^{2}}\right)$ and $\mathbf{k} \in$ $C_{b}^{1}\left(\mathbb{R}_{+}, \mathbb{R}^{N}\right)$.

Proof. The first statement is obvious since the determinant of $\mathcal{C}_{W}(a)$ is twice differentiable and bounded away from zero by uniform invertibility of $\mathcal{C}_{W}(a)$.

To prove the second statement, we note that the spectral projection onto the eigenspace associated with $\lambda=0$ is defined by

$$
\mathcal{P}(a)=(2 \pi i)^{-1} \int_{\Gamma}\left(\lambda I-\mathcal{C}_{W}(a)\right)^{-1} d \lambda,
$$

where $\Gamma$ is the circle surrounding the the eigenvalue 0 of, say, radius $\rho=-s_{C} / 2$. Then $\Gamma$ is contained in the intersection of resolvent sets of each $\mathcal{C}_{W}(a)$. Thus we can apply [13, p. 112] to claim that $\mathcal{P}(a)$ is as smooth as $\mathcal{C}_{W}$. But $\mathbf{k}$ can be expressed as $\mathbf{k}(a)=\mathcal{P}(a) \mathbf{x} /(\mathbf{x} \cdot \mathbf{1})$ for a fixed vector $\mathbf{x}$, so $\mathbf{k}$ is as smooth as $\mathcal{P}$. Since $\lambda \in \Gamma$ which is at least $-s_{C} / 2$ away from any eigenvalue of $\mathcal{C}_{W}(a), a \in \mathbb{R}_{+}$, it is clear that differentiation of (2.5) will produce bounded derivatives and hence the required derivatives of $\mathbf{k}$ are bounded.

\subsection{Lifting theorem}

While the semigroup theory, via the Duhamel formula, provides satisfactory estimates for the problem (1.1), (1.2), (1.3) with the inhomogeneity in (1.1), it is insufficient to handle inhomogenous boundary conditions $\gamma \mathbf{u}=\mathcal{B} \mathbf{u}+\mathbf{g}$ where $\mathbf{g}$ is a vector, possibly depending on time. There are various versions of trace theorems which can lift the inhomogeneity from the boundary to the interior but here the problem is complicated due to presence of the small parameter. We provide one which gives estimates uniform in $\epsilon$.

LEMMA 2.2. There is a bounded solution operator $L_{\epsilon, \lambda}: \mathbb{R}^{N} \rightarrow \mathbf{X}$ of the problem

$$
\lambda \mathbf{u}=-\mathcal{M} \mathbf{u}+\mathcal{S} \mathbf{u}+\frac{1}{\epsilon} \mathcal{C} \mathbf{u}, \quad \gamma \mathbf{u}=\mathbf{g},
$$

which satisfies $L_{\epsilon, \lambda} \mathbf{g} \in D(\mathcal{S})$ and $\left\|L_{\epsilon, \lambda}\right\| \rightarrow 0$ as $\lambda \rightarrow \infty$ uniformly in $\epsilon \in\left(0, \epsilon_{0}\right)$ for some $\epsilon_{0}>0$. 
Proof. Since $\mathcal{S}$ is the diagonal differentiation with respect to $a,(2.6)$ is just the Cauchy problem for the system of linear nonautonomous equations $\mathbf{u}_{a}=\mathcal{Q}_{\epsilon}(a) \mathbf{u}$, where $\mathcal{Q}_{\epsilon}(a):=-\lambda I-\mathcal{M}(a)+\frac{1}{\epsilon} \mathcal{C}(a)$. Since $\mathcal{Q}_{\epsilon}(a)$ is positive off-diagonal, the solution $\mathbf{u}$ is nonnegative. Let us denote by $L_{\epsilon, \lambda}(a)=\left\{l_{\epsilon, i j}(a)\right\}_{1 \leq i, j \leq N}$ the fundamental matrix of (2.6) corresponding to the unit vectors of $\mathbb{R}^{N}, \mathbf{e}_{i}=\left(\delta_{i, j}\right)_{1 \leq j \leq N}, i=1, \ldots, N$. Then $L_{\epsilon, \lambda}(a)$ is a non-negative matrix and, considered for each $a$ as the operator in $\mathbb{R}^{N}$, its $l^{1}$ norm is $\left\|L_{\epsilon, \lambda}(a)\right\|_{\mathbb{R}^{N}, 1}=\max _{1 \leq j \leq N} \sum_{i=1}^{N} l_{\epsilon, i j}(a)$. Further, for any $1 \leq j \leq N$,

$$
\frac{d}{d a} \sum_{i=1}^{N} l_{\epsilon, i j}(a)=\sum_{k=1}^{N} \sum_{i=1}^{N} q_{i k}(a) l_{\epsilon, k j}(a) \leq-\lambda \sum_{i=1}^{N} l_{\epsilon, i j}(a),
$$

since $-\mathcal{M}(a)$ is a sub-Kolmogorov matrix for each $a$. So $\sum_{i=1}^{N} l_{\epsilon, i j}(a) \leq \exp (-\lambda a)$ for each $1 \leq j \leq N$ which implies that $\left\|L_{\epsilon, \lambda}\right\|_{\leq} \lambda^{-1}$, where the latter norm is the operator norm from $\mathbb{R}^{N}$ into $\mathbf{X}$.

LEMMA 2.3. Let $\mathcal{B}$ be a bounded operator between $\mathbf{X}$ into $\mathbb{R}^{N}$. For sufficiently large $\lambda$ there is a solution operator $\mathcal{H}_{\epsilon, \lambda}: \mathbb{R}^{N} \rightarrow \mathbf{X}$ of the problem

$$
\lambda \mathbf{u}=-\mathcal{M} \mathbf{u}+\mathcal{S} \mathbf{u}+\frac{1}{\epsilon} \mathcal{C} \mathbf{u}, \quad \gamma \mathbf{u}=\mathcal{B} \mathbf{u}+\mathbf{f}
$$

with $\left\|\mathcal{H}_{\epsilon, \lambda}\right\|$ bounded independently of $\epsilon$.

Proof. Consider $L_{\epsilon, \lambda} \mathbf{g}$ for an unspecified, for a moment, vector $\mathbf{g}$. Then our problem will be solved if we can find $\mathbf{g}$ satisfying $\mathbf{g}=\mathcal{B} L_{\epsilon, \lambda} \mathbf{g}+\mathbf{f}$. Now,

$$
\left\|\mathcal{B} L_{\epsilon, \lambda} \mathbf{g}\right\|_{\mathbb{R}^{N}} \leq\|\mathcal{B}\|\left\|L_{\epsilon, \lambda} \mathbf{g}\right\|_{\mathbf{X}} \leq \lambda^{-1}\|\mathcal{B}\|\|\mathbf{g}\|_{\mathbb{R}^{N}}
$$

hence $q:=\|\mathcal{B}\|\left\|L_{\epsilon, \lambda}\right\|<1$ provided $\lambda$ is large enough. Clearly, $\lambda$ and $q$ can be chosen independently of $\epsilon$. Then $\mathbf{g}=\left(I-\mathcal{B} L_{\epsilon, \lambda}\right)^{-1} \mathbf{f}$ and, by the Neumann expansion, $\left\|\left(I-\mathcal{B} L_{\epsilon, \lambda}\right)^{-1}\right\| \leq(1-q)^{-1}$. Hence, the solution $\mathbf{u}$ to (2.7) is given by $\mathbf{u}=\mathcal{H}_{\epsilon, \lambda} \mathbf{f}=L_{\epsilon, \lambda} \mathbf{g}=L_{\epsilon, \lambda}\left(I-\mathcal{B} L_{\epsilon, \lambda}\right)^{-1} \mathbf{f}$ with $\left\|\mathcal{H}_{\epsilon, \lambda}\right\| \leq \frac{1}{\lambda(1-q)}$.

REMARK 2.4. In further applications, the boundary data $\mathbf{f}$ depends on $t$. Since the construction above does not depend on $t, \mathbf{u}$ has the same regularity in $t$ as $\mathbf{f}$ with bounds on derivatives independent of $\epsilon$. Furthermore, the operation $\left(I-\mathcal{B} L_{\epsilon, \lambda}\right)^{-1}$ acts between $\mathbb{R}^{N}$ and $\mathbb{R}^{N}$ and thus is $a$-independent. Hence, $\mathbf{u}$ is a solution of a Cauchy problem for a differential equation in $a$ and thus it is differentiable with respect to $a$.

We apply $\mathcal{H}_{\epsilon, \lambda}$ to reduce the inhomogeneous boundary problem

$$
\mathbf{u}_{t}=-\mathcal{M} \mathbf{u}+\mathcal{S} \mathbf{u}+\frac{1}{\epsilon} \mathcal{C} \mathbf{u}+\mathbf{h}, \quad \gamma \mathbf{u}=\mathcal{B} \mathbf{u}+\mathbf{f},\left.\quad \mathbf{u}\right|_{t=0}=\stackrel{\circ}{\mathbf{u}}
$$


where $\mathbf{f}$ is an $\mathbb{R}^{N}$-valued function differentiable with respect to $t$, to a problem which is homogeneous on the boundary. By introducing $\mathbf{U}=\mathbf{u}-\mathcal{H}_{\epsilon, \lambda} \mathbf{f}$, we obtain

$$
\begin{aligned}
\mathbf{U}_{t} & =\mathbf{u}_{t}-\mathcal{H}_{\epsilon, \lambda} \mathbf{f}_{t}=-\mathcal{M} \mathbf{U}+\mathcal{S} \mathbf{U}+\frac{1}{\epsilon} \mathcal{C} \mathbf{U}+\lambda \mathcal{H}_{\epsilon, \lambda} \mathbf{f}-\mathcal{H}_{\epsilon, \lambda} \mathbf{f}_{t}+\mathbf{h}, \\
\gamma \mathbf{U} & =\gamma \mathbf{u}-\gamma \mathcal{H}_{\epsilon, \lambda} \mathbf{f}=\mathcal{B} \mathbf{U}+\mathcal{B} \mathcal{H}_{\epsilon, \lambda} \mathbf{f}+\mathbf{f}-\gamma \mathcal{H}_{\epsilon, \lambda} \mathbf{f}, \\
\left.\mathbf{U}\right|_{t=0} & =\stackrel{\circ}{\mathbf{u}}-\gamma \mathcal{H}_{\epsilon, \lambda} \mathbf{f}(0) .
\end{aligned}
$$

We note that in this approach the lifting of $\mathbf{f}$ produces its time derivatives on the right hand side of the equation which creates some problems in the asymptotic analysis. This necessitates a refinement of this method which will be discussed later when we consider an integral formulation of (1.1)-(1.3).

\section{Formal asymptotic expansion}

In this section we derive formulae for the asymptotic expansion, which are formal in the sense that they are valid if all terms are smooth enough to allow for applications of necessary operations. As we noted earlier, this is not always so and a full justification of the validity of the expansion requires using integral formulation of the problem which is much more involved and is referred to the next section. However, the results given here serve as a guideline for the proper analysis and, once validated, are easier to use.

Operating formally with $\mathcal{P}$ and $\mathcal{Q}$ on both sides of (1.1) and using the fact that $\mathcal{P}$ reduces $\mathcal{C}$, we get

$$
\begin{aligned}
\mathbf{v}_{t} & =\mathcal{P} \mathcal{S P} \mathbf{v}+\mathcal{P S} \mathcal{Q} \mathbf{w}-\mathcal{P} \mathcal{M P} \mathbf{v}-\mathcal{P} \mathcal{M} \mathcal{Q} \mathbf{w} \\
\epsilon \mathbf{w}_{t} & =\varepsilon \mathcal{Q} S \mathcal{Q} \mathbf{w}+\varepsilon \mathcal{Q} S P \mathbf{v}-\epsilon \mathcal{Q} \mathcal{M P} \mathbf{v}-\epsilon \mathcal{Q} \mathcal{M} \mathbf{w}+(\mathcal{Q} C Q) \mathbf{w}, \\
\left.\mathbf{v}\right|_{t=0} & =\stackrel{\circ}{ } \stackrel{\mathbf{v}}{ },\left.\quad \mathbf{w}\right|_{t=0}=\stackrel{\circ}{\mathbf{w}},
\end{aligned}
$$

where $\stackrel{\circ}{\mathbf{v}}(a)=\mathcal{P} \stackrel{\circ}{\mathbf{n}}(a), \stackrel{\circ}{\mathbf{w}}(a)=\mathcal{Q} \stackrel{\circ}{\mathbf{n}}(a)$. Note that for symmetry of notation we use $\mathcal{P} \mathbf{n}=\mathcal{P} \mathbf{v}$ and $\mathcal{Q} \mathbf{n}=\mathcal{Q} \mathbf{w}$. Further, since $\gamma \mathbf{n}=\mathcal{P}(0) \gamma \mathbf{n}+\mathcal{Q}(0) \gamma \mathbf{n}=\gamma \mathbf{v}+\gamma \mathbf{w}$, the boundary conditions take the form

$$
\gamma \mathbf{v}=\mathcal{P}(0) \mathcal{B P} \mathbf{v}+\mathcal{P}(0) \mathcal{B} \mathcal{Q} \mathbf{w}, \quad \gamma \mathbf{w}=\mathcal{Q}(0) \mathcal{B P} \mathbf{v}+\mathcal{Q}(0) \mathcal{B} \mathcal{Q} \mathbf{w}
$$

\subsection{Projections of operators}

In the next step we shall work out explicit formulae for the projected operators.

LEMMA 3.1. For a sufficiently regular function $a \rightarrow \mathbf{n}(a)$ we have

$$
\begin{array}{ll}
{[\mathcal{P S P} \mathbf{n}](a)=-n_{a}(a) \mathbf{k}(a),} & {[\mathcal{P S} \mathcal{Q} \mathbf{n}](a)=0,} \\
{[\mathcal{Q S P} \mathbf{n}](a)=-n(a) \mathbf{k}_{a}(a),} & {[\mathcal{Q S} \mathcal{Q} \mathbf{n}](a)=-\mathbf{w}_{a}(a) .}
\end{array}
$$


Proof. By (2.1) and $\mathbf{1} \cdot \mathbf{w}=0$ for $\mathbf{w} \in W$ we get $\mathbf{1} \cdot \mathbf{k}_{a}=0, \mathbf{1} \cdot \mathbf{w}_{a}=0$, and hence $\mathbf{k}_{a}, \mathbf{w}_{a} \in W$. Next, we have $\mathcal{S P} \mathbf{n}=-(n \mathbf{k})_{a}=-n_{a} \mathbf{k}-n \mathbf{k}_{a}$ and $\mathcal{S} \mathcal{Q} \mathbf{n}=$ $\mathcal{S} \mathbf{n}-\mathcal{S P} \mathbf{n}=-\mathbf{n}_{a}+n_{a} \mathbf{k}+n \mathbf{k}_{a}$ which yields

$$
\mathcal{P} \mathcal{S P} \mathbf{n}=-\left(n_{a} \sum_{i=1}^{N} k_{i}-n \sum_{i=1}^{N} k_{i, a}\right) \mathbf{k}=-n_{a} \mathbf{k},
$$

by (2.1) and the above property of $\mathbf{k}_{a}$. Further, in a similar way

$$
\mathcal{P S} \mathbf{Q} \mathbf{n}=0, \quad \mathcal{Q} \mathcal{S P} \mathbf{n}=-n \mathbf{k}_{a},
$$

and finally, by the above property of $\mathbf{w}_{a}, \mathcal{Q S} \mathcal{Q} \mathbf{n}=-\mathbf{w}_{a}$.

To find explicit expressions for other operators appearing in (3.1) and (3.2) first, for any matrix $\mathcal{X}=\left\{x_{i j}\right\}_{1 \leq i, j \leq N}$, we denote $x^{*}:=\mathbf{1} \cdot \mathcal{X} \mathbf{k}$ and $\mathbf{x}^{\star}:=\mathcal{X} \mathbf{k}-\mu^{*} \mathbf{k}$.

LEMMA 3.2. If $\mathbf{n}$ satisfies (3.2) (or (1.2)), then

$$
\begin{aligned}
n(0) & =\gamma n=\int_{0}^{\infty} \beta^{*}(a) n(a) d a+\int_{0}^{\infty} \mathbf{1} \cdot B(a) \mathbf{w}(a) d a, \\
\gamma \mathbf{w} & =\int_{0}^{\infty} n(a) B(a) \mathbf{k}(a) d a+\int_{0}^{\infty} B(a) \mathbf{w}(a) d a-n(0) \mathbf{k}(0) \\
& =: \mathcal{B} \mathbf{v}+\mathcal{B} \mathbf{w}-\gamma \mathbf{v} .
\end{aligned}
$$

Proof. We have

$$
\begin{aligned}
& \mathcal{P}(0) \mathcal{B P} \mathbf{n}=\mathbf{k}(0) \int_{0}^{\infty} \beta^{*}(a) n(a) d a, \quad \mathcal{P}(0) \mathcal{B} \mathcal{Q} \mathbf{n}=\mathbf{k}(0) \int_{0}^{\infty} \mathbf{1} \cdot B(a) \mathbf{w}(a) d a, \\
& \mathcal{Q}(0) \mathcal{B P} \mathbf{n}=\int_{0}^{\infty} n(a) B(a) \mathbf{k}(a) d a-\mathbf{k}(0) \int_{0}^{\infty} n(a) \beta^{*}(a) d a \\
& \mathcal{Q}(0) \mathcal{B} \mathcal{Q} \mathbf{n}=\int_{0}^{\infty} B(a) \mathbf{w}(a) d a-\mathbf{k}(0) \int_{0}^{\infty} \mathbf{1} \cdot B(a) \mathbf{w}(a) d a .
\end{aligned}
$$

Then (3.3) follows from $\mathbf{k}(0) \neq 0$. Then (3.3) and $\mathbf{k}(0) n(0)=\gamma \mathbf{v}$ yield (3.4).

In a similar way we arrive at

$$
\begin{aligned}
& \mathcal{P} \mathcal{M P} \mathbf{n}=n \mu^{*} \mathbf{k}, \quad \mathcal{P} \mathcal{M} \mathcal{Q} \mathbf{n}=(\mathbf{1} \cdot \mathcal{M} \mathbf{w}) \mathbf{k}, \\
& \mathcal{Q} \mathcal{M P} \mathbf{n}=n \boldsymbol{\mu}^{\star}, \quad \mathcal{Q} \mathcal{M} \mathcal{Q} \mathbf{n}=\mathcal{M} \mathbf{w}-(\mathbf{1} \cdot \mathcal{M} \mathbf{w}) \mathbf{k}=: \mathcal{M}_{W} \mathbf{w}
\end{aligned}
$$

Using the above formulae, we can write (3.1) and (3.2) in the following more explicit form

$$
\begin{aligned}
n_{t}(t, a)= & -n_{a}(t, a)-\mu^{*}(a) n(t, a)-\mathbf{1} \cdot \mathcal{M}(a) \mathbf{w}(t, a), \\
\epsilon \mathbf{w}_{t}(t, a)= & -\epsilon \mathbf{w}_{a}(t, a)-\epsilon \mathcal{M}_{W}(a) \mathbf{w}(t, a)+\mathcal{C}_{W}(a) \mathbf{w}(t, a) \\
& -\epsilon n(t, a) \mathbf{k}_{a}(a)-\epsilon n(t, a) \boldsymbol{\mu}^{\star}(a),
\end{aligned}
$$

and

$$
\begin{aligned}
n(t, 0) & =\int_{0}^{\infty} \beta^{*}(a) n(t, a) d a+\int_{0}^{\infty} \mathbf{1} \cdot B(a) \mathbf{w}(t, a) d a, \\
\mathbf{w}(t, 0) & =\mathcal{B} \mathbf{v}(t, \cdot)+\mathcal{B} \mathbf{w}(t, \cdot)-\mathbf{v}(t, 0) .
\end{aligned}
$$




\subsection{Bulk approximation}

First we consider the bulk part approximation

$$
\mathbf{n}(t)=(\mathbf{v}(t, a), \mathbf{w}(t, a)) \approx(\overline{\mathbf{v}}(t, a), \overline{\mathbf{w}}(t, a))=\left(\overline{\mathbf{v}}(t, a), \overline{\mathbf{w}}_{0}(t, a)+\epsilon \overline{\mathbf{w}}_{1}(t, a)\right),
$$

where, as before, $\mathbf{v}=n \mathbf{k}$ and the approximate equality symbol $\approx$ accounts for the fact that we only consider the first terms of the asymptotic expansion. Following the idea of the Chapman-Enskog asymptotic method, we put $\overline{\mathbf{w}}=\overline{\mathbf{w}}_{0}+\epsilon \overline{\mathbf{w}}_{1}+\cdots$ leaving, however, $\overline{\mathbf{v}}=: \bar{n} \mathbf{k}$ unexpanded. Inserting these into (3.5), (3.6) we get:

$$
\begin{aligned}
& \bar{n}_{t}=-\bar{n}_{a}-\mu^{*} \bar{n}-\mathbf{1} \cdot \mathcal{M}\left(\overline{\mathbf{w}}_{0}+\epsilon \overline{\mathbf{w}}_{1}+\cdots\right), \\
& \overline{\mathbf{w}}_{0, t}+\epsilon \overline{\mathbf{w}}_{1, t}+\cdots=-\overline{\mathbf{w}}_{0, a}-\epsilon \overline{\mathbf{w}}_{1, a}+\cdots-\mathbf{k}_{a} \bar{n}-\boldsymbol{\mu}^{\star} \bar{n}-\mathcal{M}_{W}\left(\overline{\mathbf{w}}_{0}+\epsilon \overline{\mathbf{w}}_{1}+\cdots\right) \\
&+\frac{1}{\epsilon} \mathcal{C}_{W}\left(\overline{\mathbf{w}}_{0}+\epsilon \overline{\mathbf{w}}_{1}+\cdots\right), \\
& \gamma \bar{n}= \int_{0}^{\infty} \beta^{*} \bar{n} d a+\int_{0}^{\infty} \mathbf{1} \cdot B\left(\overline{\mathbf{w}}_{0}+\epsilon \overline{\mathbf{w}}_{1}+\cdots\right) d a \\
& \gamma \overline{\mathbf{w}}_{0}+\epsilon \gamma \overline{\mathbf{w}}_{1}+\cdots= \mathcal{B} \overline{\mathbf{v}}-\gamma \overline{\mathbf{v}}+\mathcal{B} \overline{\mathbf{w}}_{0}+\epsilon \mathcal{B} \overline{\mathbf{w}}_{1}+\cdots, \\
&\left.\bar{n}\right|_{t=0}=\stackrel{\circ}{n}=\mathbf{1} \cdot \stackrel{\circ}{\mathbf{n}},\left.\quad\left(\overline{\mathbf{w}}_{0}+\epsilon \overline{\mathbf{w}}_{1}+\cdots\right)\right|_{t=0}=\stackrel{\circ}{\mathbf{w}} .
\end{aligned}
$$

Comparing coefficients of like powers of $\epsilon$, from the second equation of (3.8) first we get $\overline{\mathbf{w}}_{0}=0$ since $\mathcal{C}_{W}$ is invertible on $W$. Next, we have

$$
\overline{\mathbf{w}}_{1}=\mathcal{C}_{W}^{-1}\left[\mathbf{k}_{a}+\boldsymbol{\mu}^{\star}\right] \bar{n} .
$$

Then, dropping $\epsilon$ order terms, we arrive at the closed system for $\bar{n}$ :

$$
\bar{n}_{t}=-\bar{n}_{a}-\mu^{*} \bar{n}, \quad \bar{n}(t, 0)=\int_{0}^{\infty} \beta^{*}(a) \bar{n}(t, a) d a, \quad \bar{n}(0, a)=\stackrel{\circ}{n},
$$

which is precisely the aggregated model (1.5), (1.6). The error of the approximation (3.7) is defined as

$$
\overline{\mathbf{E}}=(\overline{\mathbf{e}}, \overline{\mathbf{f}})=(\bar{e} \mathbf{k}, \overline{\mathbf{f}})=\left(n \mathbf{k}-\bar{n} \mathbf{k}, \mathbf{w}-\epsilon \overline{\mathbf{w}}_{1}\right) .
$$

If we assume that all terms above are sufficiently regular, then the error satisfies:

$$
\begin{aligned}
\bar{e}_{t}= & n_{t}-\bar{n}_{t}=-\bar{e}_{a}-\mu^{*} \bar{e}-\mathbf{1} \cdot \mathcal{M} \overline{\mathbf{f}}-\epsilon \mathbf{1} \cdot \mathcal{M} \overline{\mathbf{w}}_{1}, \\
\overline{\mathbf{f}}_{t}= & \mathbf{w}_{t}-\epsilon \overline{\mathbf{w}}_{1, t}=-\overline{\mathbf{f}}_{a}-\bar{e} \mathbf{k}_{a}-\bar{e} \boldsymbol{\mu}^{\star}-\mathcal{M}_{W} \overline{\mathbf{f}}+\frac{1}{\epsilon} \mathcal{C}_{W} \overline{\mathbf{f}} \\
& -\epsilon \overline{\mathbf{w}}_{1, t}-\epsilon \overline{\mathbf{w}}_{1, a}-\epsilon \mathcal{M}_{W} \overline{\mathbf{w}}_{1},
\end{aligned}
$$

with the initial conditions

$$
\left.\bar{e}\right|_{t=0}=\mathbf{0},\left.\quad \overline{\mathbf{f}}\right|_{t=0}=\stackrel{\circ}{\mathbf{w}}-\epsilon \stackrel{\circ}{n} \mathcal{C}_{W}^{-1}\left[\mathbf{k}_{a}+\mu^{\star}\right],
$$


and the boundary conditions

$$
\begin{aligned}
\gamma \bar{e} & =\int_{0}^{\infty} \beta^{*} \bar{e} d a+\int_{0}^{\infty} \mathbf{1} \cdot B \overline{\mathbf{f}} d a+\epsilon \int_{0}^{\infty} \mathbf{1} \cdot B \overline{\mathbf{w}}_{1} d a, \\
\gamma \overline{\mathbf{f}} & =\mathcal{B} \overline{\mathbf{e}}-\gamma \overline{\mathbf{e}}+\mathcal{B} \overline{\mathbf{f}}+\mathcal{B} \overline{\mathbf{v}}-\gamma \overline{\mathbf{v}}+\epsilon \mathcal{B} \overline{\mathbf{w}}_{1}-\epsilon \gamma \overline{\mathbf{w}}_{1} .
\end{aligned}
$$

Note that

$$
[\mathcal{B} \overline{\mathbf{v}}](t)=\int_{0}^{\infty} \bar{n}(t, a) B(a) \mathbf{k}(a) d a \neq \mathbf{k}(0) \int_{0}^{\infty} \bar{n}(t, a) \mathbf{1} \cdot B(a) \mathbf{k}(a) d a=\mathbf{k}(0) \bar{n}(t, 0)
$$

and thus the terms containing $\overline{\mathbf{v}}$ do not cancel out. Hence we have $O(1)$ terms both in the initial and the boundary condition and therefore we cannot hope for (3.7) being an $O(\epsilon)$ approximation of $\bar{n}$. To remedy the situation we have to introduce corrections which will take care of the transient phenomena occurring close to $t=0$ and to the boundary $a=0$. They should not 'spoil' the approximation away from spatial and temporal boundaries and thus should rapidly decrease to zero with increasing distance from both boundaries.

\subsection{Initial layer}

To construct the initial layer corrector we blow up the neighbourhood of $t=0$ by introducing the 'fast' time $\tau=t / \epsilon$ and the initial layer corrections by $\widetilde{\mathbf{n}}(\tau)=$ $(\widetilde{\mathbf{v}}(\tau), \widetilde{\mathbf{w}}(\tau))$. Thanks to the linearity of the problem, we approximate the solution $\mathbf{n}$ as the sum of the bulk part obtained above and the initial layer which we construct below. We insert the formal expansion

$$
\widetilde{\mathbf{v}}(\tau, a)=\widetilde{\mathbf{v}}_{0}(\tau, a)+\epsilon \widetilde{\mathbf{v}}_{1}(\tau, a)+\cdots, \quad \widetilde{\mathbf{w}}(\tau, a)=\widetilde{\mathbf{w}}_{0}(\tau, a)+\epsilon \widetilde{\mathbf{w}}_{1}(\tau, a)+\cdots
$$

into the system (3.1) getting, for $\widetilde{\mathbf{v}}_{i}=\widetilde{n}_{i} \mathbf{k}, i=0,1$,

$$
\begin{aligned}
\epsilon^{-1}\left(\widetilde{n}_{0, \tau}+\epsilon \widetilde{n}_{1, \tau}+\cdots\right)= & -\widetilde{n}_{0, a}-\epsilon \widetilde{n}_{1, a} \cdots-\mu^{*}\left(\widetilde{n}_{0}+\epsilon \widetilde{n}_{1}+\cdots\right) \\
& -\mathbf{1} \cdot \mathcal{M}\left(\widetilde{\mathbf{w}}_{0}+\epsilon \widetilde{\mathbf{w}}_{1}+\cdots\right), \\
\epsilon^{-1}\left(\widetilde{\mathbf{w}}_{0, \tau}+\epsilon \widetilde{\mathbf{w}}_{1, \tau}+\cdots\right)= & -\widetilde{\mathbf{w}}_{0, a}-\epsilon \widetilde{\mathbf{w}}_{1, a}-\cdots-\left(\widetilde{n}_{0}+\epsilon \widetilde{n}_{1}+\cdots\right) \mathbf{k}_{a} \\
& -\left(\widetilde{n}_{0}+\epsilon \widetilde{n}_{1}+\cdots\right) \boldsymbol{\mu}^{\star}-\mathcal{M}{ }_{W}\left(\widetilde{\mathbf{w}}_{0}+\epsilon \widetilde{\mathbf{w}}_{1}+\cdots\right) \\
& +\frac{1}{\epsilon} \mathcal{C}_{W}\left(\widetilde{\mathbf{w}}_{0}+\widetilde{\mathbf{w}}_{1}+\cdots\right) \\
\gamma\left(\widetilde{n}_{0}+\epsilon \widetilde{n}_{1}+\cdots\right)= & \int_{0}^{\infty} \beta^{*}\left(\widetilde{n}_{0}+\epsilon \widetilde{n}_{1}+\cdots\right) d a \\
& +\int_{0}^{\infty} \mathbf{1} \cdot B\left(\widetilde{\mathbf{w}}_{0}+\epsilon \widetilde{\mathbf{w}}_{1}+\cdots\right) d a \\
\gamma\left(\widetilde{\mathbf{w}}_{0}+\epsilon \widetilde{\mathbf{w}}_{1}+\cdots\right)= & \mathcal{B}\left(\widetilde{\mathbf{v}}_{0}+\epsilon \widetilde{\mathbf{v}}_{1}+\cdots\right)-\gamma\left(\widetilde{\mathbf{v}}_{0}+\epsilon \widetilde{\mathbf{v}}_{1}+\cdots\right) \\
& +\mathcal{B}\left(\widetilde{\mathbf{w}}_{0}+\epsilon \widetilde{\mathbf{w}}_{1}+\cdots\right), \\
\left.\widetilde{n}\right|_{t=0}= & 0,\left.\quad\left(\widetilde{\mathbf{w}}_{0}+\epsilon \widetilde{\mathbf{w}}_{1}+\cdots\right)\right|_{t=0}=\stackrel{\mathbf{w}}{ }
\end{aligned}
$$


where in the initial condition we have taken into account that the exact initial condition for the hydrodynamic part is already satisfied by the bulk hydrodynamic approximation but the bulk kinetic part cannot satisfy the exact initial condition.

Comparing coefficients at like powers of $\epsilon$, from the first equation we immediately obtain $\widetilde{n}_{0, \tau}=0$ which implies $\widetilde{n}_{0}$ on account of the decay to zero of the initial layer term. Then, at the same level, we obtain $\widetilde{\mathbf{w}}_{0, \tau}=\mathcal{C}_{W}(a) \widetilde{\mathbf{w}}_{0}$ which yields

$$
\widetilde{\mathbf{w}}_{0}=e^{\tau \mathcal{C}_{W}(a)} \stackrel{\circ}{\mathbf{w}}
$$

where $a$ is a parameter. We note that due to the assumption that $\lambda=0$ is the dominant eigenvalue of $\mathcal{C}(a)$ uniformly in $a$, the type of $\left(e^{\tau \mathcal{C}_{W}(a)}\right)_{\tau \geq 0}$ in $W$ is negative uniformly in $a$ and thus $\widetilde{\mathbf{w}}_{0}(\tau)$ decays to 0 exponentially fast. We also note that the initial layer is fully determined by the initial condition $\stackrel{\circ}{\mathbf{w}}$ and thus no corrections to the boundary conditions can be made at this level; on the contrary, as we shall see, the initial layer introduces an additional error on the boundary.

We modify the approximation (3.7) taking into account the initial layer:

$$
(\mathbf{v}(t, a), \mathbf{w}(t, a)) \approx\left(\overline{\mathbf{v}}(t, a), \epsilon \overline{\mathbf{w}}_{1}(t, a)+\widetilde{\mathbf{w}}_{0}(t / \epsilon, a)\right)
$$

and define the new error

$$
\begin{aligned}
\widetilde{\mathbf{E}}(t, a) & =(\widetilde{\mathbf{e}}(t, a), \widetilde{\mathbf{f}}(t, a))=(\widetilde{e}(t, a) \mathbf{k}, \widetilde{\mathbf{f}}(t, a)) \\
& =\left(\mathbf{v}(t, a)-\overline{\mathbf{v}}(t, a), \mathbf{w}(t, a)-\epsilon \overline{\mathbf{w}}_{1}(t, a)-\widetilde{\mathbf{w}}_{0}(t / \epsilon, a)\right) \\
& =\left(\overline{\mathbf{e}}(t, a), \overline{\mathbf{f}}(t, a)-\widetilde{\mathbf{w}}_{0}(t / \epsilon, a)\right) .
\end{aligned}
$$

Again, assuming that all terms are sufficiently smooth and using linearity of the problem, we get from (3.12)

$$
\begin{aligned}
\widetilde{e}_{t}= & -\widetilde{e}_{a}-\mu^{*} \widetilde{e}-\mathbf{1} \cdot \mathcal{M} \widetilde{\mathbf{f}}-\epsilon \boldsymbol{\mu} \cdot \overline{\mathbf{w}}_{1}-\mathbf{1} \cdot \mathcal{M} \widetilde{\mathbf{w}}_{0}, \\
\widetilde{\mathbf{f}}_{t}= & -\widetilde{\mathbf{f}}_{a}-\widetilde{e} \mathbf{k}_{a}-\widetilde{e} \boldsymbol{\mu}^{\star}-\mathcal{M}{ }_{W} \widetilde{\mathbf{f}}+\frac{1}{\epsilon} \mathcal{C}_{W} \widetilde{\mathbf{f}} \\
& -\epsilon \overline{\mathbf{w}}_{1, t}-\epsilon \overline{\mathbf{w}}_{1, a}-\epsilon \mathcal{M}_{W} \overline{\mathbf{w}}_{1}-\widetilde{\mathbf{w}}_{0, a}-\mathcal{M}_{W} \widetilde{\mathbf{w}}_{0} .
\end{aligned}
$$

Similarly, we get

$$
\left.\widetilde{e}\right|_{\tau=0}=0,\left.\quad \widetilde{\mathbf{f}}\right|_{\tau=0}=-\epsilon \stackrel{\circ}{n} \mathcal{C}_{W}^{-1}\left[\mathbf{k}_{a}+\mu^{\star}\right]
$$

and

$$
\begin{aligned}
& \gamma \widetilde{e}=\int_{0}^{\infty} \beta^{*} \widetilde{e} d a+\int_{0}^{\infty} \mathbf{1} \cdot B \widetilde{\mathbf{f}} d a+\epsilon \int_{0}^{\infty} \mathbf{1} \cdot B \overline{\mathbf{w}}_{1} d a+\int_{0}^{\infty} \mathbf{1} \cdot B \widetilde{\mathbf{w}}_{0} d a, \\
& \gamma \widetilde{\mathbf{f}}=\mathcal{B} \widetilde{\mathbf{e}}-\gamma \widetilde{\mathbf{e}}+\mathcal{B} \widetilde{\mathbf{f}}+\mathcal{B} \overline{\mathbf{v}}-\gamma \overline{\mathbf{v}}+\epsilon \mathcal{B} \overline{\mathbf{w}}_{1}+\mathcal{B} \widetilde{\mathbf{w}}_{0}-\epsilon \gamma \overline{\mathbf{w}}_{1}-\gamma \widetilde{\mathbf{w}}_{0} .
\end{aligned}
$$

As expected, the troublesome $O(1)$ term $\mathcal{B} \overline{\mathbf{v}}-\gamma \overline{\mathbf{v}}$ in the boundary condition has been unaffected by the initial layer. Also the initial layer has introduced a new short range error at $a=0$. This necessitates introduction of the boundary layer. 


\subsection{Boundary layer}

The boundary layer is constructed by blowing up the state variable $a$ according to $\alpha=a / \epsilon$ and defining

$$
\hat{\mathbf{n}}(t, \alpha)=(\hat{\mathbf{v}}(t, \alpha), \hat{\mathbf{w}}(t, \alpha))
$$

The operator $\mathcal{S}$ is a first order differentiation operator, hence the change of variables $a \rightarrow \alpha=a / \epsilon$ gives

$$
\mathcal{S}_{a} \hat{\mathbf{n}}=\frac{1}{\epsilon} \mathcal{S}_{\alpha} \hat{\mathbf{n}}
$$

where the subscripts denote the variable which $\mathcal{S}$ acts on.

Again, the linearity allows to approximate the solution $\mathbf{n}$ by the sum of the bulk and initial layer parts, obtained above, and the boundary layer:

$$
\hat{\mathbf{v}}(t, \alpha)=\hat{\mathbf{v}}_{0}(t, \alpha)+\epsilon \hat{\mathbf{v}}_{1}(t, \alpha)+\cdots, \quad \hat{\mathbf{w}}(t, \alpha)=\hat{\mathbf{w}}_{0}(t, \alpha)+\epsilon \hat{\mathbf{w}}_{1}(t, \alpha)+\cdots
$$

We insert the expansion into (3.1) and, repeating the procedure of the previous section, we get that at the zeroth level the boundary layer is given by

$$
\hat{n}_{0, \alpha}=0, \quad-\widehat{\mathbf{w}}_{0, \alpha}+\mathcal{C}_{W}(0) \widehat{\mathbf{w}}_{0}=0
$$

which is simply the stationary original equation with coefficients frozen at $a=0$ : $\mathcal{S}_{\alpha} \hat{\mathbf{n}}+\mathcal{C}(0) \hat{\mathbf{n}}=0$, and we are free to chose the boundary conditions which will help to eliminate the term $\mathcal{B} \overline{\mathbf{v}}-\gamma \overline{\mathbf{v}}$. To find it, let us assume that we have a solution to the above equation with, for a moment, unspecified boundary condition and, as before, define the new approximation

$$
\begin{aligned}
\mathbf{n}(t, a)= & (n(t, a) \mathbf{k}(a), \mathbf{w}(t, a)) \approx\left(\bar{n}(t, a)+\hat{n}(t, a / \epsilon), \epsilon \overline{\mathbf{w}}_{1}(t, a)\right. \\
& \left.+\tilde{\mathbf{w}}_{0}(t / \epsilon, a)+\hat{\mathbf{w}}(t, a / \epsilon)\right) .
\end{aligned}
$$

It follows that we can take $\widehat{n}_{0}(t, \alpha) \equiv 0$. Let us define the new error

$$
\widehat{\mathbf{E}}(t, a)=(\hat{e}(t, a) \mathbf{k}(a), \hat{\mathbf{f}}(t, a))=\left(\tilde{e}(t, a) \mathbf{k}(a), \tilde{\mathbf{f}}(t, a)-\hat{\mathbf{w}}_{0}(t, a / \epsilon)\right)
$$

Then

$$
\begin{aligned}
\hat{e}_{t}= & \tilde{e}_{t}-\widehat{v}_{0, t}=-\widehat{e}_{a}-\mu^{*} \widehat{e}-\mathbf{1} \cdot \mathcal{M} \widehat{\mathbf{f}}-\mathbf{1} \cdot \mathcal{M} \hat{\mathbf{w}}_{0}-\epsilon \mathbf{1} \cdot \mathcal{M} \overline{\mathbf{w}}_{1}-\mathbf{1} \cdot \mathcal{M} \widetilde{\mathbf{w}}_{0}, \\
\widehat{\mathbf{f}}_{t}= & \widetilde{\mathbf{f}}_{t}-\widehat{\mathbf{w}}_{0, t}=-\widehat{\mathbf{f}}_{a}-\widehat{e} \mathbf{k}_{a}-\widehat{e} \boldsymbol{\mu}^{\star}-\mathcal{M} \widehat{\mathbf{f}}_{W}+\frac{1}{\epsilon} \mathcal{C}_{W} \hat{\mathbf{f}}-\epsilon \overline{\mathbf{w}}_{1, t}-\epsilon \overline{\mathbf{w}}_{1, a}-\epsilon \mathcal{M}{ }_{W} \overline{\mathbf{w}}_{1} \\
& -\widetilde{\mathbf{w}}_{0, a}-\mathcal{M}_{W} \widetilde{\mathbf{w}}_{0}-\mathcal{M}_{W} \widehat{\mathbf{w}}_{0}+\frac{1}{\epsilon}\left(\mathcal{C}_{W}-\mathcal{C}_{W}(0)\right) \widehat{\mathbf{w}}_{0}-\widehat{\mathbf{w}}_{0, t},
\end{aligned}
$$


and for the boundary conditions we obtain

$$
\begin{aligned}
\gamma \widehat{e}= & \gamma \widetilde{e}=\int_{0}^{\infty} \beta^{*} \widehat{e} d a+\int_{0}^{\infty} \mathbf{1} \cdot B \widehat{\mathbf{f}} d a+\epsilon \int_{0}^{\infty} \mathbf{1} \cdot B \overline{\mathbf{w}}_{1} d a \\
& +\int_{0}^{\infty} \mathbf{1} \cdot B \widetilde{\mathbf{w}}_{0} d a+\int_{0}^{\infty} \mathbf{1} \cdot B \widehat{\mathbf{w}}_{0} d a \\
\gamma \widehat{\mathbf{f}}= & \gamma \widetilde{\mathbf{f}}-\gamma \widehat{\mathbf{w}}_{0}=\mathcal{B} \widehat{\mathbf{e}}-\gamma \widehat{\mathbf{e}}+\mathcal{B} \widehat{\mathbf{f}}+\mathcal{B} \overline{\mathbf{v}}-\gamma \overline{\mathbf{v}} \\
& +\epsilon \mathcal{B} \overline{\mathbf{w}}_{1}+\mathcal{B} \widetilde{\mathbf{w}}_{0}-\epsilon \gamma \overline{\mathbf{w}}_{1}-\gamma \widetilde{\mathbf{w}}_{0}+\mathcal{B} \widehat{\mathbf{w}}_{0}-\gamma \widehat{\mathbf{w}}_{0} .
\end{aligned}
$$

Thus, to eliminate the bulk term on the boundary, the boundary layer should be the solution to

$$
\widehat{\mathbf{w}}_{0, \alpha}=\mathcal{C}_{W}(0) \widehat{\mathbf{w}}_{0}, \quad \widehat{\mathbf{w}}_{0}(0)=\mathcal{B} \overline{\mathbf{v}}-\gamma \overline{\mathbf{v}}
$$

which is just a system of linear equations with constant coefficients (and with a parameter $t$ entering through the initial condition). We note that the right hand side of the second equation in (3.22) satisfies

$$
[\mathbf{1} \cdot(\mathcal{B} \overline{\mathbf{v}}-\gamma \overline{\mathbf{v}})](t)=\int_{0}^{\infty} \beta^{*}(a) \bar{n}(t, a) d a-\bar{n}(t, 0)(\mathbf{1} \cdot \mathbf{k}(0))=0,
$$

by (3.10) and the normalization of $\mathbf{k}$, and hence (3.22) is consistent in the sense that both sides are in $W$.

The initial conditions for system (3.21) take the following form:

$$
\left.\hat{e}\right|_{t=0}=0,\left.\quad \widehat{\mathbf{f}}\right|_{t=0}=-\hat{\mathbf{w}}_{0}(0, a / \epsilon)-\epsilon \overline{\mathbf{w}}_{1}(0, a) .
$$

We note that, even with the boundary layer, we still have terms depending on $t / \epsilon$ which, when lifted as in (2.8) will, upon differentiation with respect to $t$, produce $O(1 / \epsilon)$ terms on the right hand side. This necessitates introduction of the corner layer.

\subsection{Corner layer}

As noted above, the boundary terms which depend on $t / \epsilon$ give rise to an $\epsilon$ order error. To eliminate this initial layer contribution on the boundary, we need to introduce the corner layer by simultaneously rescaling time and space: $\tau=t / \epsilon, \alpha=a / \epsilon$. As before we use linearity and seek the corner layer independently by inserting the formal expansion

$$
\breve{\mathbf{v}}(\tau, \alpha)=\breve{\mathbf{v}}_{0}(\tau, \alpha)+\epsilon \breve{\mathbf{v}}_{1}(\tau, \alpha)+\cdots, \quad \breve{\mathbf{w}}(\tau, \alpha)=\breve{\mathbf{w}}_{0}(\tau, \alpha)+\epsilon \breve{\mathbf{w}}_{1}(\tau, \alpha)+\cdots
$$

into the system (3.1). Following the procedure for the initial layer we get

$$
\breve{n}_{0, \tau}=-\breve{n}_{0, \alpha}, \quad \breve{\mathbf{w}}_{0, \tau}=-\breve{\mathbf{w}}_{0, \alpha}+\mathcal{C}_{W}(0) \breve{\mathbf{w}}_{0},
$$

which is the unperturbed original equation in $(\tau, \alpha)$-variables with coefficients frozen at $a=0: \breve{\mathbf{n}}_{0, \tau}=\mathcal{S}_{\alpha} \breve{\mathbf{n}}_{0}+\mathcal{C}(0) \breve{\mathbf{n}}_{0}$. Hence, here we have freedom of choosing 
both the boundary and the initial conditions (in $(\tau, \alpha)$-variables) which will help to eliminate the problematic terms on the boundary. To find the proper side conditions, let us assume that we have a solution to the above equation with, for the moment, unspecified boundary condition and, as before, define the new approximation

$$
\begin{aligned}
\mathbf{n}(t, a)=(\mathbf{v}(t, a), \mathbf{w}(t, a)) \approx & (\overline{\mathbf{v}}(t, a)+\breve{\mathbf{v}}(t / \epsilon, a / \epsilon), \\
& \left.\epsilon \overline{\mathbf{w}}_{1}(t, a)+\tilde{\mathbf{w}}_{0}(t / \epsilon, a)+\hat{\mathbf{w}}(t, a / \epsilon)+\breve{\mathbf{w}}(t / \epsilon, a / \epsilon)\right),
\end{aligned}
$$

with the error of this approximation given by

$$
\breve{\mathbf{E}}(t, a)=(\breve{\mathbf{e}}(t, a), \breve{\mathbf{f}}(t, a))=\left(\hat{\mathbf{e}}(t, a)-\breve{\mathbf{v}}_{0}(t / \epsilon, a / \epsilon), \widehat{\mathbf{f}}(t, a)-\breve{\mathbf{w}}_{0}(t / \epsilon, a / \epsilon)\right) .
$$

Following the procedure described for the boundary layer, we find that to eliminate the $O(1)$ entries in the equation for the error on the boundary we have to impose the following boundary conditions for (3.23)

$$
\gamma \breve{n}_{0}=\int_{0}^{\infty} \mathbf{1} \cdot B \widetilde{\mathbf{w}}_{0} d a, \quad \gamma \breve{\mathbf{w}}_{0}=\mathcal{B} \widetilde{\mathbf{w}}_{0}-\gamma \widetilde{\mathbf{w}}_{0}-\gamma\left(\breve{n}_{0} \mathbf{k}\right) .
$$

Also, as for the boundary layer, we find that the second equation of (3.24) is properly posed in $W$. We complement the problem for the corner layer by the homogeneous initial conditions: $\left.\breve{n}_{0}\right|_{\tau=0}=\left.\breve{\mathbf{w}}_{0}\right|_{\tau=0}=0$.

Taking all layers into account, we find that the final error formally satisfies

$$
\begin{aligned}
& \breve{\mathbf{E}}_{t}=\mathcal{S} \breve{\mathbf{E}}-\mathcal{M} \breve{\mathbf{E}}+\frac{1}{\epsilon} \mathcal{C} \breve{\mathbf{E}} \\
& -\left[\begin{array}{c}
\mathbf{1} \cdot \mathcal{M} \widetilde{\mathbf{w}}_{0} \\
\widetilde{\mathbf{w}}_{0, a}+\mathcal{M}_{W} \widetilde{\mathbf{w}}_{0}
\end{array}\right]-\epsilon\left[\begin{array}{c}
1 \cdot \mathcal{M} \overline{\mathbf{w}}_{1} \\
\overline{\mathbf{w}}_{1, t}+\overline{\mathbf{w}}_{1, a}+\mathcal{M}_{W} \overline{\mathbf{w}}_{1}
\end{array}\right] \\
& -\left[\begin{array}{c}
\mathbf{1} \cdot \mathcal{M} \hat{\mathbf{w}}_{0} \\
\mathcal{M}_{W} \widehat{\mathbf{w}}_{0}-\epsilon^{-1}\left(\mathcal{C}_{W}-\mathcal{C}_{W}(0)\right) \widehat{\mathbf{w}}_{0}+\widehat{\mathbf{w}}_{0, t}
\end{array}\right] \\
& -\left[\begin{array}{c}
\mu^{*} \breve{n}_{0}+\mathbf{1} \cdot \mathcal{M} \breve{\mathbf{w}}_{0} \\
\breve{n}_{0} \mathbf{k}_{a}+\breve{n}_{0} \boldsymbol{\mu}^{\star}+\mathcal{M}_{W} \breve{\mathbf{w}}_{0}-\epsilon^{-1}\left(\mathcal{C}_{W}-\mathcal{C}_{W}(0)\right) \breve{\mathbf{w}}_{0}
\end{array}\right], \\
& \gamma \breve{\mathbf{E}}=\mathcal{B} \breve{\mathbf{E}}+\epsilon\left[\begin{array}{c}
\int_{0}^{\infty} \mathbf{1} \cdot B \overline{\mathbf{w}}_{1} d a \\
\mathcal{B} \overline{\mathbf{w}}_{1}-\gamma \overline{\mathbf{w}}_{1}
\end{array}\right]+\left[\begin{array}{c}
\int_{0}^{\infty} \mathbf{1} \cdot B \widehat{\mathbf{w}}_{0} d a \\
\mathcal{B} \widehat{\mathbf{w}}_{0}
\end{array}\right] \\
& +\left[\begin{array}{c}
\int_{0}^{\infty} \beta^{*} \breve{n}_{0} d a+\int_{0}^{\infty} 1 \cdot B \breve{\mathbf{w}}_{0} d a \\
\mathcal{B} \breve{\mathbf{v}}_{0}+\mathcal{B} \breve{\mathbf{w}}_{0}
\end{array}\right], \\
& \left.\breve{\mathbf{E}}\right|_{t=0}=\left[-\hat{\mathbf{w}}_{0}(0, a / \epsilon)-\epsilon \overline{\mathbf{w}}_{1}(0, a)\right] .
\end{aligned}
$$

However, as we emphasized a few times, for (3.25) to be valid, the solution $\mathbf{n}$ and all terms of the asymptotic expansion must be strongly differentiable with respect to $t$ and belong to the domain of the generator which, as mentioned in the introduction, equals $\left\{\mathbf{u} \in W_{1}^{1}\left(\mathbb{R}_{+}\right) ; \mathbf{u}(0)=\mathcal{B} \mathbf{u}\right\}$. This is not always easy to achieve. In fact, in general an initial condition $\stackrel{\circ}{\mathbf{n}}$ which satisfies $\stackrel{\circ}{\mathbf{n}}(0)=\mathcal{B} \stackrel{\circ}{\mathbf{n}}$, will not satisfy the condition $\stackrel{\circ}{n}=\mathbf{1} \cdot \stackrel{\circ}{\mathbf{n}}=\int_{0}^{\infty} \beta^{*}(a) \stackrel{\circ}{n}(a) d a$, required for differentiability of the solution of the aggregated problem. 


\section{Integral formulation}

It turns out that we have to work with mild solutions of the equations. To set the stage, let us consider our population model (1.1) in a more compact form:

$$
\begin{aligned}
\mathbf{u}_{t}(t, a) & =-\mathbf{u}_{a}(t, a)+\mathcal{K}[\mathbf{u}(t, \cdot)], \\
\mathbf{u}(0, a) & =\stackrel{\circ}{\mathbf{u}}(a), \quad \mathbf{u}(t, 0)=\mathcal{B}[\mathbf{u}(t, \cdot)] .
\end{aligned}
$$

The operators $\mathcal{K}: \mathbf{X} \rightarrow \mathbf{X}$ and $\mathcal{B}: \mathbf{X} \rightarrow \mathbb{R}^{N}$ are linear and bounded.

System (4.1) can be reduced to an integral equation by integration along characteristics. It turns out that the solution of this integral equation defines the semigroup generated by the operator $\mathcal{A} \mathbf{u}=-\mathbf{u}_{a}+\mathcal{K} \mathbf{u}$ on the domain $D(\mathcal{A})=$ $\left\{\mathbf{u} \in W_{1}^{1}\left(\mathbb{R}_{+}\right) ; \mathbf{u}(0)=\mathcal{B} \mathbf{u}\right\}$. Precisely speaking, let us consider the integral equation obtained by integrating (4.1) along the characteristics $a-t=$ constant:

$$
\mathbf{u}(t, a)= \begin{cases}\stackrel{\circ}{\mathbf{u}}(a-t)+\int_{0}^{t} \mathcal{K}[\mathbf{u}(\tau, \cdot)](\tau+a-t) d \tau, & a>t, \\ \mathcal{B}[\mathbf{u}(t-a, \cdot)]+\int_{t-a}^{t} \mathcal{K}[\mathbf{u}(\tau, \cdot)](\tau+a-t) d \tau, & a<t,\end{cases}
$$

where here and below the notation $a<t$ and $a>t$ are understood as the respective inequality almost everywhere. Then the family of operators defined as $[\mathcal{G}(t) \stackrel{\circ}{\mathbf{u}}](a):=$ $\mathbf{u}(t, a)$, where $\mathbf{u}(t, a)$ is the solution of (4.2) with $\stackrel{0}{\mathbf{u}} \in \mathbf{X}$ is the semigroup on $\mathbf{X}$ generated by $(\mathcal{A}, D(\mathcal{A}))$, see [19, (1.49), Propositions 3.2 and 3.7].

In the error estimates we shall need mild solutions of the inhomogeneous problem associated with (4.1):

$$
\mathbf{u}_{t}(t, a)=-\mathbf{u}_{a}(t, a)+\mathcal{K}[\mathbf{u}(t, \cdot)]+\mathbf{f}(t, a),
$$

with the same initial and boundary conditions as in (4.1), where $t \rightarrow \mathbf{f}(t)$ is a function from $(0, \infty)$ to $\mathbf{X}$. However, (4.3) does not make sense if $\mathbf{u}$ is not differentiable which, in turn, cannot be achieved unless $\stackrel{\circ}{\mathbf{u}} \in D(\mathcal{A})$ and $\mathbf{f}$ is an $\mathbf{X}$-differentiable, or a $D(\mathcal{A})$-continuous, function. In general, we have to work with mild solutions of (4.3) defined by

$$
\mathbf{u}(t)=\mathcal{G}(t) \stackrel{\circ}{\mathbf{u}}+\int_{0}^{t} \mathcal{G}(t-s) \mathbf{f}(s) d s .
$$

This definition is not very helpful as it views $(\mathcal{G}(t))_{t \geq 0}$ somewhat globally without noticing the structure visible in (4.2). However, we can prove the following result:

PROPOSITION 4.1. A function $\mathbf{u} \in C\left(\mathbb{R}_{+}, \mathbf{X}\right)$ is a mild solution of (4.3) if and only if

$$
\mathbf{u}(t, a)= \begin{cases}\stackrel{\circ}{\mathbf{u}}(a-t)+\int_{0}^{t} \mathcal{K}[\mathbf{u}(\sigma, \cdot)](\sigma+a-t) d \sigma+\int_{0}^{t} \mathbf{f}(\sigma, \sigma+a-t) d \sigma, & a>t, \\ \mathcal{B}[\mathbf{u}(t-a, \cdot)]+\int_{t-a}^{t} \mathcal{K}[\mathbf{u}(\sigma, \cdot)](\sigma+a-t) d \sigma+\int_{t-a}^{t} \mathbf{f}(\sigma, \sigma+a-t) d \sigma, & a<t .\end{cases}
$$


Proof. First, to shorten the notation, we denote, for arbitrary numbers $a, \sigma, t$,

$$
\sigma_{a, t}=(\sigma, \sigma+a-t)
$$

and, for any $a$-dependent operation $A$ and a function $(t, a) \rightarrow u(t, a)$ we denote $A[u(t, \cdot)](a)=[A u](t, a)$ (or $[A u](t)$ if the output is $a$-independent).

It can be proved, [4, Proposition 3.31], that a function $\mathbf{u} \in C\left(\mathbb{R}_{+}, \mathbf{X}\right)$ is a mild solution to (4.3) with $\mathbf{f} \in L_{1}\left(\mathbb{R}_{+}, \mathbf{X}\right)$ if and only if $\int_{0}^{t} \mathbf{u}(s) d s \in D(\mathcal{A})$ and

$$
\mathbf{u}(t)=\stackrel{\circ}{\mathbf{u}}+\mathcal{A} \int_{0}^{t} \mathbf{u}(s) d s+\int_{0}^{t} \mathbf{f}(s) d s, \quad t \geq 0 .
$$

Hence, $\mathbf{u}$ is a mild solution to (4.3) if and only if $\mathbf{v}(t):=\int_{0}^{t} \mathbf{u}(s) d s \in D(\mathcal{A})$ is the classical solution to

$$
\mathbf{v}_{t}(t, a)=\stackrel{\circ}{\mathbf{u}}(a)-\mathbf{v}_{a}(t, a)+\mathcal{K}[\mathbf{v}(t, \cdot)](a)+\mathbf{F}(t, a),
$$

with $\mathbf{v}(0, a)=0$ and $\mathbf{v}(t, 0)=\mathcal{B}[\mathbf{v}(t, \cdot)]$, where $\mathbf{F}(t, a)=\int_{0}^{t} \mathbf{f}(\tau, a) d \tau$. Eqs. (4.7) are satisfied pointwise and thus we can integrate them along characteristics

$$
\mathbf{v}(t, a)=\left\{\begin{array}{cc}
\int_{0}^{t} \mathcal{K}[\mathbf{v}(\tau, \cdot)](\tau+a-t) d \tau+\int_{0}^{t} \mathbf{F}\left(\tau_{a, t}\right) d \tau+\int_{0}^{t} \stackrel{\circ}{\mathbf{u}}(\tau+a-t) d \tau, & a>t, \\
\mathcal{B}[\mathbf{v}(t-a, \cdot)]+\int_{t-a}^{t} \mathcal{K}[\mathbf{v}(\tau, \cdot)](\tau+a-t) d \tau+\int_{t-a}^{t} \mathbf{F}\left(\tau_{a, t}\right) d \tau & \\
+\int_{t-a}^{t} \mathbf{u}(\tau+a-t) d \tau, & a<t .
\end{array}\right.
$$

Then, by changing the order of integration and changing variables in respective terms, we find

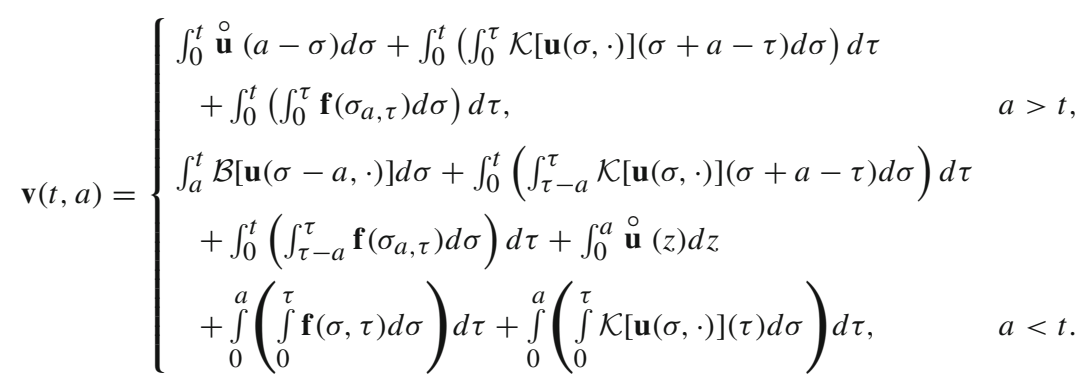

and, using $\mathbf{v}(t, a)=\int_{0}^{t} \mathbf{u}(\sigma, a) d \sigma$, upon differentiation we arrive at (4.5).

Various terms of the asymptotic expansion appear in a direct form which is incompatible with (4.2) and must be re-written to allow for accommodation into the integral formulation.

As in Lemma 2.2, for sufficiently large $\lambda$ there is a classical solution of the stationary problem

$$
\lambda \mathbf{w}=-\mathbf{w}_{a}+\mathcal{K} \mathbf{w}, \quad \gamma \mathbf{w}=\mathcal{B} \mathbf{w}+\mathbf{g},
$$


where $\mathbf{g}$ may depend on $t>0$. Moreover, $\mathbf{w}$ is differentiable with respect to $a$ (as a solution to a system of ODEs) and with respect to $t$ provided $\mathbf{g}(t)$ is differentiable.

Since Eq. (4.10) is satisfied pointwise, we can integrate it along characteristics to obtain

$$
\begin{aligned}
\lambda \int_{0}^{t} \mathbf{w}\left(\sigma_{a, t}\right) d \sigma & =-\int_{0}^{t} \mathbf{w}, 2\left(\sigma_{a, t}\right) d \sigma+\int_{0}^{t}[\mathcal{K} \mathbf{w}]\left(\sigma_{a, t}\right) d \sigma, \quad a>t, \\
\lambda \int_{t-a}^{t} \mathbf{w}\left(\sigma_{a, t}\right) d \sigma & =-\int_{t-a}^{t} \mathbf{w}, 2\left(\sigma_{a, t}\right) d \sigma+\int_{t-a}^{t}[\mathcal{K} \mathbf{w}]\left(\sigma_{a, t}\right) d \sigma, \quad a<t,
\end{aligned}
$$

where $\mathbf{w},{ }_{i}$ denotes the partial derivative with respect to the $i$-th variable. Now,

$$
\frac{\partial}{\partial \sigma} \mathbf{w}\left(\sigma_{a, t}\right)=\mathbf{w}, 1\left(\sigma_{a, t}\right)+\mathbf{w}, 2\left(\sigma_{a, t}\right)
$$

and therefore, integrating with respect to $\sigma$ from 0 to $t$, we obtain

$$
\begin{aligned}
& \mathbf{w}(t, a)-\mathbf{w}(0, a-t)=\int_{0}^{t} \mathbf{w},_{1}\left(\sigma_{a, t}\right) d \sigma+\int_{0}^{t} \mathbf{w}, 2\left(\sigma_{a, t}\right) d \sigma, \quad a>t, \\
& \mathbf{w}(t, a)-\mathbf{w}(t-a, 0)=\int_{t-a}^{t} \mathbf{w},_{1}\left(\sigma_{a, t}\right) d \sigma+\int_{t-a}^{t} \mathbf{w}, 2\left(\sigma_{a, t}\right) d \sigma, \quad a<t .
\end{aligned}
$$

Combining these with (4.11) we obtain

$\mathbf{w}(t, a)=\left\{\begin{array}{cc}\mathbf{w}(0, a-t)+\int_{0}^{t}[\mathcal{K} \mathbf{w}]\left(\sigma_{a, t}\right) d \sigma+\int_{0}^{t} \mathbf{w},{ }_{1}\left(\sigma_{a, t}\right) d \sigma-\lambda \int_{0}^{t} \mathbf{w}\left(\sigma_{a, t}\right) d \sigma, & a>t, \\ {[\mathcal{B} \mathbf{w}](t-a)+\mathbf{g}(t-a)+\int_{t-a}^{t}[\mathcal{K} \mathbf{w}]\left(\sigma_{a, t}\right) d \sigma+\int_{t-a}^{t} \mathbf{w},{ }_{1}\left(\sigma_{a, t}\right) d \sigma} & \\ -\lambda \int_{t-a}^{t} \mathbf{w}\left(\sigma_{a, t}\right) d \sigma, & a<t .\end{array}\right.$

It turns out that the inhomogeneous boundary data are better treated separately. By linearity, we can consider the case with $\stackrel{\circ}{\mathbf{u}}=0$ and $\mathbf{f}(t)=0$.

Denote by $\mathcal{V}_{\mathcal{K}}$ the fundamental solution matrix of the equation $\mathbf{z}_{a}^{\prime}(a)=\mathcal{K}(a) \mathbf{z}(a)$; that is, $\mathbf{z}(a)=\mathcal{V}_{\mathcal{K}}(a) \mathbf{z}_{0}$ satisfies the above equation with $\mathbf{z}(0)=\mathbf{z}_{0}$, see e.g., $\left[16\right.$, p. 242]. We recall that in our considerations $\mathcal{K}=L_{\epsilon, 0}=-\mathcal{M}+\epsilon^{-1} \mathcal{C}$ and, by Lemma 2.2 with $\lambda=0$,

$$
\sup _{a \in \mathbb{R}_{+}}\left\|\mathcal{V}_{\mathcal{K}}(a)\right\|_{\mathbb{R}^{N}, 1} \leq 1
$$

LEMMA 4.2. Assume that, in addition to assumptions of this section, $\mathcal{K}$ satisfies (4.12) and let $\mathbf{g} \in C\left([0, \infty), \mathbb{R}^{N}\right)$. Then $\mathbf{u}$ is a continuous solution to

$$
\mathbf{u}(t, a)= \begin{cases}\int_{0}^{t} \mathcal{K}[\mathbf{u}(\tau, \cdot)](\tau+a-t) d \tau, & a>t, \\ \mathcal{B}[\mathbf{u}(t-a, \cdot)]+\mathbf{g}(t-a)+\int_{t-a}^{t} \mathcal{K}[\mathbf{u}(\tau, \cdot)](\tau+a-t) d \tau, & a<t,\end{cases}
$$

if and only if

$$
\mathbf{u}(t, a)=\mathcal{V}_{\mathcal{K}}(a) \omega(t, a)
$$


where

$$
\boldsymbol{\omega}(t, a)= \begin{cases}0, & a>t, \\ \left(\left(I-\mathcal{B} \mathcal{V}_{\mathcal{K}}\right)^{-1} \mathbf{g}\right)(t-a), & a<t .\end{cases}
$$

Proof. For regular $\mathbf{g}$ we can re-write the problem as a differential equation (satisfied in each triangle $t<a$ and $t>a$ ) and, using invertibility of $\mathcal{V}_{\mathcal{K}}$, we see that $\omega$ defined by (4.14) satisfies

$$
\boldsymbol{\omega}(t, a)= \begin{cases}0, & a>t, \\ \mathcal{B} \mathcal{V}_{\mathcal{K}}[\omega(t-a, \cdot)]+\mathbf{g}(t-a), & a<t .\end{cases}
$$

The solution $\omega$ of this problem is given by the solution of the simple problem

$$
\omega(t, a)= \begin{cases}0, & a>t, \\ \psi(t-a), & a<t .\end{cases}
$$

provided $\boldsymbol{\psi}(t)=\mathcal{B} \mathcal{V}_{\mathcal{K}}[\boldsymbol{\psi}(t-\cdot)]+\mathbf{g}(t)$. This is a Volterra equation which, considered in $C\left([0, T], \mathbb{R}^{N}\right)$ for any fixed $T<+\infty$, can be solved using standard Picard iterations yielding a unique solution

$$
\boldsymbol{\psi}(t)=\left[\left(I-\mathcal{B} \mathcal{V}_{\mathcal{K}}\right)^{-1} \mathbf{g}\right](t)
$$

with $\left\|\left(I-\mathcal{B} \mathcal{V}_{\mathcal{K}}\right)^{-1}\right\|_{C\left([0, T], \mathbb{R}^{N}\right)} \leq e^{m T}$, where $m=\sup _{s \in[0, T]}\left\|B(s) \mathcal{V}_{\mathcal{K}}(s)\right\|_{\mathbb{R}^{N}}$. Let us take a sequence of $W_{1}^{1}$ functions $\mathbf{g}_{n}$ converging uniformly on $[0, T]$ to a continuous function $\mathbf{g}$. Then $\boldsymbol{\psi}_{n}=\left[\left(I-\mathcal{B} \mathcal{V}_{\mathcal{K}}\right)^{-1} \mathbf{g}_{n}\right]$ converges uniformly on $[0, T]$ to

$$
\boldsymbol{\psi}=\left[\left(I-\mathcal{B} \mathcal{V}_{\mathcal{K}}\right)^{-1} \mathbf{g}\right]
$$

as $\left(I-\mathcal{B} \mathcal{V}_{\mathcal{K}}\right)^{-1}$ is a continuous operator on $C\left([0, T], \mathbb{R}^{N}\right)$ (in fact, on $L_{\infty}([0, T)$, $\left.\mathbb{R}^{N}\right)$ ). Thus,

$$
\omega_{n}(t, a)= \begin{cases}0, & t<a<T, \\ \psi_{n}(t-a), & 0<a<t,\end{cases}
$$

converges uniformly on $[0, T] \times[0, T]$ to $\omega$ given by $(4.15)$ and hence $\mathcal{V}_{K}(a) \omega_{n}(t, a)$ uniformly converges to a continuous function on $[0, t] \times[0, T]$ and to zero on $(t, \infty) \times$ $[0, T]$; we denote the limit by $\overline{\mathbf{u}}(t, a)$. Clearly $\overline{\mathbf{u}}(t, a)$ is a solution of (4.13) as all operators in (4.13) are bounded. Moreover $\overline{\mathbf{u}}(t, a)$ treated as a function $t \rightarrow \overline{\mathbf{u}}(t, \cdot)$ is in $C\left([0, T], L_{1}\left(\mathbb{R}_{+}\right)\right)$by

$$
\begin{aligned}
\int_{0}^{\infty}\|\overline{\mathbf{u}}(t+h, a)-\overline{\mathbf{u}}(t, a)\|_{\mathbb{R}^{N}} d a= & \int_{0}^{t}\|\overline{\mathbf{u}}(t+h, a)-\overline{\mathbf{u}}(t, a)\|_{\mathbb{R}^{N}} d a \\
& +\int_{t}^{t+h}\|\overline{\mathbf{u}}(t+h, a)\|_{\mathbb{R}^{N}} d a
\end{aligned}
$$

and the uniform continuity of $\overline{\mathbf{u}}(t, a)$ as a function of two variables in the triangle $[0, t] \times[0, T]$. But the difference of two solutions to (4.13) satisfies its homogeneous version (with $\mathbf{g}=0$ ) for which we can use the semigroup theory which, see e.g. [19, Theorem 2.1], ensures the uniqueness. Hence, the only solution to (4.13) with continuous $\mathbf{g}$ is given by (4.14). The reverse statement follows similarly by applying $\mathcal{V}_{\mathcal{K}}$ to the equation satisfied by $\omega$. 


\section{Error estimates}

As we noted, in general it is impossible to have differentiable solutions of all the problems involved in the construction of the asymptotic expansion. Thus we have to rewrite the error system (3.25) in the form of the integrated Eq. (4.5). The mild solutions of (1.1) in the projected form (3.3), (3.5) satisfy:

$$
n(t, a)=\left\{\begin{array}{cl}
\stackrel{\circ}{n}(a-t)-\int_{0}^{t}\left[\mu^{*} n\right]\left(\sigma_{a, t}\right) d \sigma-\int_{0}^{t}[\mathbf{1} \cdot \mathcal{M} \mathbf{w}]\left(\sigma_{a, t}\right) d \sigma, & a>t \\
\int_{0}^{\infty} n(t-a, s) \beta^{*}(s) d s+\int_{0}^{\infty} \mathbf{1} \cdot B(s) \mathbf{w}(t-a, s) d s & \\
-\int_{t-a}^{t}\left[\mu^{*} n\right]\left(\sigma_{a, t}\right) d \sigma-\int_{t-a}^{t}[\mathbf{1} \cdot \mathcal{M} \mathbf{w}]\left(\sigma_{a, t}\right) d \sigma, & a<t,
\end{array}\right.
$$

and

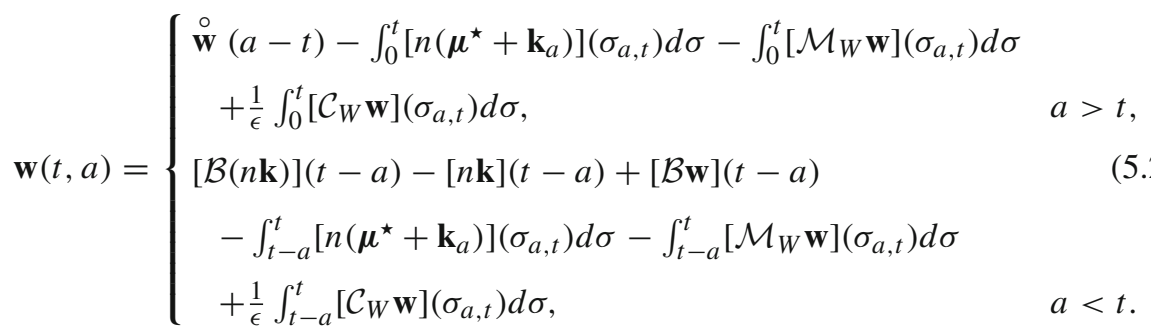

In the same manner, the solution of the aggregated Eq. (3.10) satisfies

$$
\bar{n}(t, a)= \begin{cases}\stackrel{\circ}{n}(a-t)-\int_{0}^{t}\left[\mu^{*} \bar{n}\right]\left(\sigma_{a, t}\right) d \sigma, & a>t \\ \int_{0}^{\infty} \bar{n}(t-a, s) \beta^{*}(s) d s-\int_{t-a}^{t}\left[\mu^{*} \bar{n}\right]\left(\sigma_{a, t}\right) d \sigma, & a<t .\end{cases}
$$

The system above is a one-dimensional version of (4.2). Using [19, Theorem 2.9] or [12, Theorem 4.3], we have that the cohort functions $\sigma \rightarrow \bar{n}(\sigma, \xi), \xi=a-t$, are continuously differentiable with respect to $\sigma$ for all $\xi<0$ and almost all $\xi>0$, with

$$
\frac{d}{d \sigma} \bar{n}(\sigma, \sigma+\xi)=-\mu(\sigma+\xi) \bar{n}(\sigma, \sigma+\xi) .
$$

In the next step we write the kinetic part of the bulk expansion $\overline{\mathbf{w}}_{1}=\mathcal{C}_{W}^{-1}\left[\mathbf{k}_{a}+\boldsymbol{\mu}^{\star}\right] \bar{n}$ (see (3.9)) in the integrated form. Using the time derivative of the cohort function, we have

$$
\begin{aligned}
& \overline{\mathbf{w}}_{1}(t, a)-\overline{\mathbf{w}}(0, a-t)=\int_{0}^{t} \frac{d}{d \sigma} \overline{\mathbf{w}}_{1}\left(\sigma_{a, t}\right) d \sigma, \quad a>t, \\
& \overline{\mathbf{w}}_{1}(t, a)-\overline{\mathbf{w}}_{1}(t-a, 0)=\int_{t-a}^{t} \frac{d}{d \sigma} \overline{\mathbf{w}}_{1}\left(\sigma_{a, t}\right) d \sigma, \quad a<t .
\end{aligned}
$$

But, by (5.4) and Lemma 2.1,

$$
\begin{aligned}
\frac{d}{d \sigma} \overline{\mathbf{w}}_{1}\left(\sigma_{a, t}\right) & =\frac{d}{d \sigma}\left(\mathcal{C}_{W}^{-1}(\sigma+a-t)\left[\mathbf{k}_{a}(\sigma+a-t)+\mu^{\star}(\sigma+a-t)\right] \bar{n}\left(\sigma_{a, t}\right)\right) \\
& =\Upsilon(\sigma+a-t) \bar{n}\left(\sigma_{a, t}\right),
\end{aligned}
$$


where the function $\Upsilon$ is bounded, again by Lemma 2.1 and assumptions on $\mathcal{M}$. In what follows we denote

$$
\boldsymbol{\gamma}_{W}(a)=\mathcal{C}_{W}^{-1}(a)\left[\mathbf{k}_{a}(a)+\boldsymbol{\mu}^{\star}(a)\right] .
$$

Hence

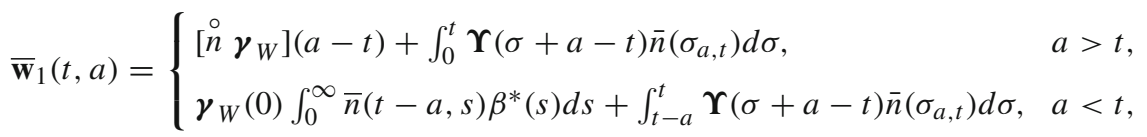

where we used the initial condition $\bar{n}(t-a, 0)=\int_{0}^{\infty} \bar{n}(t-a, s) \beta^{*}(s) d s$.

In the next step we write the initial layer (3.16) in the integrated form. For this we note that (3.16) is of the same form as (4.10) if we introduce $\widetilde{\mathbf{w}}_{\epsilon}(t, a)=\widetilde{\mathbf{w}}_{0}(\tau, a)$ and put $\lambda=0, \mathcal{K}=\frac{1}{\epsilon} \mathcal{C}_{W}$ and $\mathcal{B}=0$ (and with $t$ and $a$ variables interchanged); that is

$\widetilde{\mathbf{w}}_{\epsilon}(t, a)= \begin{cases}\stackrel{\circ}{\mathbf{w}}(a-t)+\frac{1}{\epsilon} \int_{0}^{t}\left[\mathcal{C}_{W} \widetilde{\mathbf{w}}_{\epsilon}\right]\left(\sigma_{a, t}\right) d \sigma+\int_{0}^{t} \widetilde{\mathbf{w}}_{\epsilon, 2}\left(\sigma_{a, t}\right) d \sigma, & a>t, \\ \exp \left(\frac{t-a}{\epsilon} \mathcal{C}_{W}(0)\right) \stackrel{\circ}{\mathbf{w}}(0)+\frac{1}{\epsilon} \int_{t-a}^{t}\left[\mathcal{C}_{W} \widetilde{\mathbf{w}}_{\epsilon}\right]\left(\sigma_{a, t}\right) d \sigma+\int_{t-a}^{t} \widetilde{\mathbf{w}}_{\epsilon, 2}\left(\sigma_{a, t}\right) d \sigma, & a<t .\end{cases}$

In the same way for the boundary layer $\widehat{\mathbf{w}}_{\epsilon}(t, a)=\widehat{\mathbf{w}}_{0}(t, \alpha)$ we obtain the representation

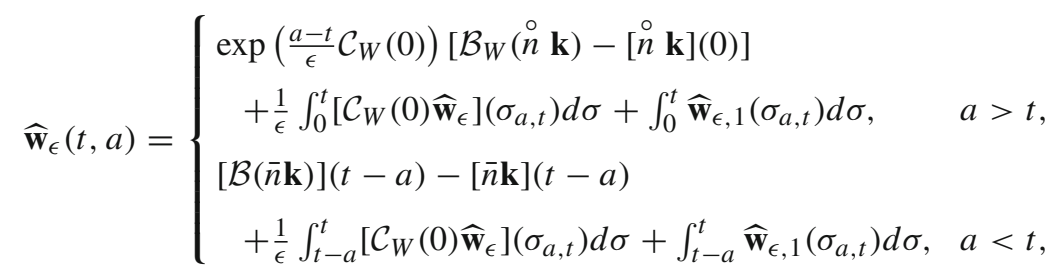

where, since $\stackrel{\circ}{\mathbf{n}} \in W_{1}^{1}\left(\mathbb{R}_{+}\right)$, the value $\stackrel{\circ}{n}(0)$ is well-defined and $\mathcal{B}(\overline{\mathbf{v}}) \rightarrow \mathcal{B}(\stackrel{\circ}{n} \mathbf{k})$ for $t \rightarrow 0^{+}$as $\mathcal{B}$ is bounded and $\overline{\mathbf{v}}=\bar{n} \mathbf{k}, \bar{n}$ being a continuous in $t, \mathbf{X}$-valued solution to $(5.1)$.

Finally, we find the integral representation of the corner layer. The corner layer solves the equation of the same type as the original equation so there is no need to perform any additional transformations. However, it is clear that the boundary conditions (3.24) are not compatible at $\alpha=\tau=0$ with the homogeneous initial conditions and thus the problem must be considered in the integrated form.

First let us note that the equations in (3.23) are decoupled. The problem for $\breve{n}_{0}$ is of the form

$$
\breve{n}_{0, \tau}(\tau, \alpha)=-\breve{n}_{0, \alpha}(\tau, \alpha), \quad \breve{n}_{0}(0, \alpha)=0, \quad \breve{n}_{0}(\tau, 0)=F(\tau),
$$

where $\left.F(\tau)=\int_{0}^{\infty} \mathbf{1} \cdot B e^{\tau \mathcal{C}_{W}(a)} \stackrel{\circ}{\mathbf{w}}(a)\right] d a$. Hence,

$$
\breve{n}_{0}(\tau, \alpha)= \begin{cases}0, & \tau<\alpha, \\ \int_{0}^{\infty}\left[\mathbf{1} \cdot B e^{(\tau-\alpha) \mathcal{C}_{W}(a)} \stackrel{\circ}{\mathbf{w}}(a)\right] d a, & \tau>\alpha .\end{cases}
$$


The kinetic part of the corner layer $\breve{\mathbf{w}}_{0}$ satisfies

$$
\breve{\mathbf{w}}_{0}(\tau, \alpha)= \begin{cases}\int_{0}^{\tau}\left[\mathcal{C}_{W}(0) \breve{\mathbf{w}}_{0}\right]\left(\sigma_{\alpha, \tau}\right) d \sigma, & \tau<\alpha, \\ \mathbf{F}(\tau-\alpha)+\int_{\tau-\alpha}^{\tau}\left[\mathcal{C}_{W}(0) \breve{\mathbf{w}}_{0}\right]\left(\sigma_{\alpha, \tau}\right) d \sigma, & \tau>\alpha\end{cases}
$$

where, recall, $\sigma_{\alpha, \tau}=(\sigma, \sigma+\alpha-\tau)$ and

$$
\mathbf{F}(\tau)=\int_{0}^{\infty} B(a) \widetilde{\mathbf{w}}_{0}(\tau, a) d a-\widetilde{\mathbf{w}}_{0}(\tau, 0)-\mathbf{k}(0) \int_{0}^{\infty} \mathbf{1} \cdot B(a) \widetilde{\mathbf{w}}_{0}(\tau, a) d a .
$$

We note that (5.9) can be simplified as in Lemma 4.2. In this case the the fundamental solution matrix of the equation $\mathbf{z}_{a}^{\prime}(a)=\mathcal{C}_{W}(0) \mathbf{z}(a)$ is simply the matrix exponential: $\mathbf{z}(a)=e^{a \mathcal{C}_{W}(0)}$. Using the fact that the initial value is 0 and $\mathcal{B}=0$ we immediately obtain

$$
\breve{\mathbf{w}}_{0}(\tau, \alpha)= \begin{cases}0, & \tau<\alpha \\ e^{\alpha \mathcal{C}_{W}(0)} \mathbf{F}(\tau-\alpha), & \tau>\alpha\end{cases}
$$

To simplify notation, let

$$
\begin{array}{ll}
\widetilde{\mathbf{w}}_{0, \epsilon}(t, a)=\widetilde{\mathbf{w}}_{0}(\tau, a), & \widehat{\mathbf{w}}_{0, \epsilon}(t, a)=\widehat{\mathbf{w}}_{0}(t, \alpha), \\
\breve{\mathbf{w}}_{0, \epsilon}(t, a)=\breve{\mathbf{w}}_{0}(\tau, \alpha), & \breve{n}_{0, \epsilon}(t, a)=\breve{n}_{0}(\tau, \alpha) .
\end{array}
$$

Combining the above we arrive at the following equations of the error in the integrated form:

(i) for the aggregated ('hydrodynamic') part and $a>t$ :

$$
\begin{aligned}
\breve{e}(t, a)= & -\int_{0}^{t}\left[\mu^{*} \breve{e}\right]\left(\sigma_{a, t}\right) d \sigma-\int_{0}^{t}[\mathbf{1} \cdot \mathcal{M} \breve{\mathbf{f}}]\left(\sigma_{a, t}\right) d \sigma \\
& -\int_{0}^{t}\left[\mathbf{1} \cdot \mathcal{M}\left(\epsilon \overline{\mathbf{w}}_{1}+\widetilde{\mathbf{w}}_{0, \epsilon}+\widehat{\mathbf{w}}_{0, \epsilon}+\breve{\mathbf{w}}_{0, \epsilon}\right)\right]\left(\sigma_{a, t}\right) d \sigma
\end{aligned}
$$

where we used the fact that $\breve{n}_{0}=0$ for $t<a$;

(ii) for the aggregated part and $a<t$ :

$$
\begin{aligned}
\breve{e}(t, a)= & \int_{0}^{\infty} \breve{e}(t-a, s) \beta^{*}(s) d s+\int_{0}^{\infty} \mathbf{1} \cdot B(s) \breve{\mathbf{f}}(t-a, s) d s-\int_{t-a}^{t}\left[\mu^{*} \breve{e}\right]\left(\sigma_{a, t}\right) d \sigma \\
& -\int_{t-a}^{t}[\mathbf{1} \cdot \mathcal{M} \breve{\mathbf{f}}]\left(\sigma_{a, t}\right) d \sigma+\int_{0}^{\infty} \breve{n}_{0, \epsilon}(t-a, s) \beta^{*}(s) d s \\
& +\int_{0}^{\infty} \mathbf{1} \cdot B(s)\left(\epsilon \overline{\mathbf{w}}_{1}(t-a, s)+\widehat{\mathbf{w}}_{0, \epsilon}(t-a, s)+\breve{\mathbf{w}}_{0, \epsilon}(t-a, s)\right) d s \\
& -\int_{t-a}^{t}\left[\mu^{*} \breve{n}_{0, \epsilon}\right]\left(\sigma_{a, t}\right) d \sigma-\int_{t-a}^{t}\left[\mathbf{1} \cdot \mathcal{M}\left(\epsilon \overline{\mathbf{w}}_{1}+\widetilde{\mathbf{w}}_{0, \epsilon}+\widehat{\mathbf{w}}_{0, \epsilon}+\breve{\mathbf{w}}_{0, \epsilon}\right)\right]\left(\sigma_{a, t}\right) d \sigma,
\end{aligned}
$$


(iii) for the complementary ('kinetic') part and $a>t$ :

$$
\begin{aligned}
\breve{\mathbf{f}}(t, a)= & -\epsilon\left[\stackrel{\circ}{n} \boldsymbol{\gamma}_{W}\right](a-t)-\exp \left(\frac{a-t}{\epsilon} \mathcal{C}_{W}(0)\right)[\mathcal{B}(\stackrel{\circ}{ } \mathbf{k})-\stackrel{\circ}{n} \mathbf{k}(0)] \\
& -\int_{0}^{t}\left[\breve{e}\left(\boldsymbol{\mu}^{\star}+\mathbf{k}_{a}\right)\right]\left(\sigma_{a, t}\right) d \sigma-\int_{0}^{t}\left[\mathcal{M}_{W} \breve{\mathbf{f}}\right]\left(\sigma_{a, t}\right) d \sigma+\frac{1}{\epsilon} \int_{0}^{t}\left[\mathcal{C}_{W} \breve{\mathbf{f}}\right]\left(\sigma_{a, t}\right) d \sigma \\
& -\int_{0}^{t}\left[\breve{n}_{0}\left(\boldsymbol{\mu}^{\star}+\mathbf{k}_{a}\right)\right]\left(\sigma_{a, t}\right) d \sigma-\int_{0}^{t}\left[\mathcal{M}_{W}\left(\epsilon \overline{\mathbf{w}}_{1}+\widetilde{\mathbf{w}}_{0, \epsilon}+\widehat{\mathbf{w}}_{0, \epsilon}+\breve{\mathbf{w}}_{0, \epsilon}\right)\right]\left(\sigma_{a, t}\right) d \sigma \\
& -\epsilon \int_{0}^{t} \boldsymbol{\Upsilon}(\sigma+a-t) \bar{n}\left(\sigma_{a, t}\right) d \sigma+\frac{1}{\epsilon} \int_{0}^{t}\left[\left(\mathcal{C}_{W}-\mathcal{C}_{W}(0)\right) \widehat{\mathbf{w}}_{0, \epsilon}\right]\left(\sigma_{a, t}\right) d \sigma \\
& +\frac{1}{\epsilon} \int_{0}^{t}\left[\left(\mathcal{C}_{W}-\mathcal{C}_{W}(0)\right) \breve{\mathbf{w}}_{0, \epsilon}\right]\left(\sigma_{a, t}\right) d \sigma-\int_{0}^{t} \widetilde{\mathbf{w}}_{0, \epsilon, 2}\left(\sigma_{a, t}\right) d \sigma-\int_{0}^{t} \widehat{\mathbf{w}}_{\epsilon, 1}\left(\sigma_{a, t}\right) d \sigma,
\end{aligned}
$$

(iv) for the complementary ('kinetic') part and $t>a$ :

$$
\begin{aligned}
\breve{\mathbf{f}}(t, a)= & {[\mathcal{B}(\breve{e} \mathbf{k})](t-a)-[\gamma(\breve{e} \mathbf{k})](t-a)+[\mathcal{B} \mathbf{f}](t-a)+\left[\mathcal{B}\left(\breve{n}_{0, \epsilon} \mathbf{k}\right)\right](t-a) } \\
& +\left[\mathcal{B}\left(\epsilon \overline{\mathbf{w}}_{1}+\widehat{\mathbf{w}}_{0, \epsilon}+\breve{\mathbf{w}}_{0, \epsilon}\right)\right](t-a)-\epsilon \boldsymbol{\gamma}_{W}(0) \int_{0}^{\infty} \bar{n}(t-a, s) \beta^{*}(s) d s \\
& -\int_{t-a}^{t}\left[\breve{e}\left(\boldsymbol{\mu}^{\star}+\mathbf{k}_{a}\right)\right]\left(\sigma_{a, t}\right) d \sigma-\int_{t-a}^{t}\left[\mathcal{M}_{W} \breve{\mathbf{f}}\right]\left(\sigma_{a, t}\right) d \sigma+\frac{1}{\epsilon} \int_{t-a}^{t}\left[\mathcal{C}_{W} \breve{\mathbf{f}}\right]\left(\sigma_{a, t}\right) d \sigma \\
& -\int_{t-a}^{t}\left[\breve{n}_{0, \epsilon}\left(\boldsymbol{\mu}^{\star}+\mathbf{k}_{a}\right)\right]\left(\sigma_{a, t}\right) d \sigma-\int_{t-a}^{t}\left[\mathcal{M}_{W}\left(\epsilon \overline{\mathbf{w}}_{1}+\widetilde{\mathbf{w}}_{0, \epsilon}+\widehat{\mathbf{w}}_{0, \epsilon}+\breve{\mathbf{w}}_{0, \epsilon}\right)\right]\left(\sigma_{a, t}\right) d \sigma \\
& +\frac{1}{\epsilon} \int_{t-a}^{t}\left[\left(\mathcal{C}_{W}-\mathcal{C}_{W}(0)\right)\left(\widehat{\mathbf{w}}_{0, \epsilon}+\breve{\mathbf{w}}_{0, \epsilon}\right)\right]\left(\sigma_{a, t}\right) d \sigma-\epsilon \int_{t-a}^{t} \mathbf{\Upsilon}(\sigma+a-t) \bar{n}\left(\sigma_{a, t}\right) d \sigma \\
& -\int_{t-a}^{t} \widetilde{\mathbf{w}}_{0, \epsilon, 2}\left(\sigma_{a, t}\right) d \sigma-\int_{t-a}^{t} \widehat{\mathbf{w}}_{0, \epsilon, 1}\left(\sigma_{a, t}\right) d \sigma
\end{aligned}
$$

where in (iii) and (iv) we used the change of variables of the type

$$
\int_{t / \epsilon-a / \epsilon}^{t / \epsilon}\left[\mathcal{C}_{W}(0) \breve{\mathbf{w}}_{0}\right]\left(\sigma_{a / \epsilon, t / \epsilon}\right) d \sigma=\frac{1}{\epsilon} \int_{t-a}^{t}\left[\mathcal{C}_{W}(0) \breve{\mathbf{w}}_{0, \epsilon}\right]\left(\sigma_{a, t}^{\prime}\right) d \sigma^{\prime}
$$

The initial condition of the error $\breve{\mathbf{E}}(0, a)=(\breve{\mathbf{e}}(0, a), \breve{\mathbf{f}}(0, a))$ is thus

$$
\breve{\mathbf{E}}(0, a)=\left[\begin{array}{c}
0 \\
-\epsilon\left[\stackrel{\circ}{n} \gamma_{W}\right](a)-\exp \left(\mathcal{C}_{W}(0) \frac{a}{\epsilon}\right)[\mathcal{B}(\stackrel{\circ}{n} \mathbf{k})-\stackrel{\circ}{n} \mathbf{k}(0)]
\end{array}\right],
$$


the inhomogeneity in the equation is given by

$$
\begin{aligned}
\breve{\mathbf{F}}(t, a)= & -\left[\begin{array}{c}
\mathbf{1} \cdot \mathcal{M}(a) \widetilde{\mathbf{w}}_{0}\left(\frac{t}{\epsilon}, a\right) \\
\widetilde{\mathbf{w}}_{0, a}\left(\frac{t}{\epsilon}, a\right)+\mathcal{M}_{W}(a) \widetilde{\mathbf{w}}_{0}\left(\frac{t}{\epsilon}, a\right)
\end{array}\right] \\
- & \epsilon\left[\begin{array}{c}
\mathbf{1} \cdot \mathcal{M}(a) \overline{\mathbf{w}}_{1}(t, a) \\
\boldsymbol{\Upsilon}(a) \bar{n}(t, a)+\mathcal{M}_{W}(a) \overline{\mathbf{w}}_{1}(t, a)
\end{array}\right] \\
& -\left[\begin{array}{c}
\mathbf{1} \cdot \mathcal{M}(a) \hat{\mathbf{w}}_{0}\left(t, \frac{a}{\epsilon}\right) \\
\mathcal{M}_{W}(a) \widehat{\mathbf{w}}_{0}\left(t, \frac{a}{\epsilon}\right)-\epsilon^{-1}\left(\mathcal{C}_{W}(a)-\mathcal{C}_{W}(0)\right) \widehat{\mathbf{w}}_{0}\left(t, \frac{a}{\epsilon}\right)+\widehat{\mathbf{w}}_{0, t}\left(t, \frac{a}{\epsilon}\right)
\end{array}\right] \\
& -\left[\begin{array}{c}
\mu^{*}(a) \breve{n}_{0}\left(\frac{t}{\epsilon}, \frac{a}{\epsilon}\right)+\mathbf{1} \cdot \mathcal{M}(a) \breve{\mathbf{w}}_{0}\left(\frac{t}{\epsilon}, \frac{a}{\epsilon}\right) \\
\left.\breve{n}_{0}, \frac{a}{\epsilon}\right) \mathbf{k}_{a}(a)+\breve{n}_{0}\left(\frac{t}{\epsilon}, \frac{a}{\epsilon}\right) \boldsymbol{\mu}^{\star}(a)+\mathcal{M}_{W}(a) \breve{\mathbf{w}}_{0}\left(\frac{t}{\epsilon}, \frac{a}{\epsilon}\right) \\
\left.-\epsilon^{-1}\left(\mathcal{C}_{W}(a)-\mathcal{C}_{W}(0)\right) \breve{\mathbf{w}}_{0}\left(\frac{t}{\epsilon}, \frac{a}{\epsilon}\right)\right)
\end{array}\right] \\
=: & \mathbf{F}_{1}\left(\frac{t}{\epsilon}, a\right)+\mathbf{F}_{2}(t, a)+\mathbf{F}_{3}\left(t, \frac{a}{\epsilon}\right)+\mathbf{F}_{4}\left(\frac{t}{\epsilon}, \frac{a}{\epsilon}\right),
\end{aligned}
$$

which is similar to (3.25) but for $\overline{\mathbf{w}}_{1, t}+\overline{\mathbf{w}}_{1, a}$ which has been replaced, thanks to (5.5), by the term $\Upsilon \bar{n}$ which requires lower regularity from the data. Finally, the inhomogeneity on the boundary is given by

$$
\mathbf{g}(t)=\left[\begin{array}{c}
\int_{0}^{\infty} \breve{n}_{0, \epsilon}(t, s) \beta^{*}(s) d s+\int_{0}^{\infty} \mathbf{1} \cdot B(s)\left(\epsilon \overline{\mathbf{w}}_{1}(t, s)+\widehat{\mathbf{w}}_{0, \epsilon}(t, s)+\breve{\mathbf{w}}_{0, \epsilon}(t, s)\right) d s \\
\left(\left[\mathcal{B}\left(\breve{n}_{0, \epsilon}(t, \cdot) \mathbf{k}(\cdot)\right)\right]+\left[\mathcal{B}\left(\epsilon \mathbf{w}_{1}(t, \cdot)+\widehat{\mathbf{w}}_{0, \epsilon}(t, \cdot)+\breve{\mathbf{w}}_{0, \epsilon}(t, \cdot)\right)\right]\right. \\
\left.-\epsilon \boldsymbol{\gamma}_{W}(0) \int_{0}^{\infty} \bar{n}(t, s) \beta^{*}(s) d s\right)
\end{array}\right],
$$

where we recall that

$$
\begin{aligned}
\overline{\mathbf{w}}_{1}(t, 0) & =\left[\mathcal{C}_{W}^{-1}(0)\left(\mathbf{k}_{a}(0)+\boldsymbol{\mu}^{\star}(0)\right)\right] \int_{0}^{\infty} \bar{n}(t, s) \beta^{*}(s) d s \\
& =\boldsymbol{\gamma}_{W}(0) \int_{0}^{\infty} \bar{n}(t, s) \beta^{*}(s) d s .
\end{aligned}
$$

THEOREM 5.1. Let us assume that $\mathcal{C}, B$ and $\mathcal{M}$ satisfy assumptions introduced in Sect. 2.1 and $\mathbf{n}_{\epsilon}(t, a):=\left[\mathcal{G}_{\epsilon}(t) \stackrel{\circ}{\mathbf{n}}\right](a)=n_{\epsilon}(t, a) \mathbf{k}(a)+\mathbf{w}_{\epsilon}(t, a)$ be a solution to (1.1). Then, for each $T<\infty$ there exists a constant $C(T, \mathcal{M}, B, \mathcal{C})$ such that for any $\stackrel{\circ}{\mathbf{n}} \in W_{1}^{1}\left(\mathbb{R}_{+}, \mathbb{R}^{N}\right)$ and uniformly on $[0, T]$ we have

$$
\begin{gathered}
\|n(t, \cdot)-\bar{n}(t, \cdot)\|_{L_{1}\left(\mathbb{R}_{+}\right)} \leq \epsilon C(T, \mathcal{M}, B, \mathcal{C})\|\stackrel{\circ}{\mathbf{n}}\|_{W_{1}^{1}\left(\mathbb{R}_{+}, \mathbb{R}^{N}\right)}, \\
\left\|\mathbf{w}(t, \cdot)-e^{\frac{t}{\epsilon} \mathcal{C}(\cdot)} \stackrel{\circ}{\mathbf{w}}(\cdot)\right\|_{L_{1}\left(\mathbb{R}_{+}, \mathbb{R}^{N}\right)} \leq \epsilon C(T, \mathcal{M}, B, \mathcal{C})\|\stackrel{\circ}{\mathbf{n}}\|_{W_{1}^{1}\left(\mathbb{R}_{+}, \mathbb{R}^{N}\right)} .
\end{gathered}
$$

Proof. We use linearity and first estimate the part of the error, denoted by $\breve{\mathbf{E}}_{1}$, coming from $\breve{\mathbf{F}}$ and the initial condition (5.16) with $\mathbf{g}=0$ using the semigroup formula (4.4) and then we let the initial conditions and $\breve{\mathbf{F}}$ equal to zero and use (4.14) to estimate the part of the error $\breve{\mathbf{E}}_{2}$ due to the nonzero $\mathbf{g}$. 
Let us recall that the semigroup $\left(\mathcal{G}_{\epsilon}(t)\right)_{t \geq 0}$ generated by the system (1.1) is equibounded in $\epsilon:\left\|\mathcal{G}_{\epsilon}(t)\right\| \leq \exp \omega t$ with $\omega$ independent of $\epsilon$. By [19, (1.49), Propositions 3.2 and 3.7] and (5.12)-(5.15), $\breve{\mathbf{E}}_{1}$ satisfies

$$
\breve{\mathbf{E}}_{1}(t)=\mathcal{G}_{\epsilon}(t) \breve{\mathbf{E}}(0, \cdot)+\int_{0}^{t} \mathcal{G}_{\epsilon}(t-s) \breve{\mathbf{F}}(s) d s .
$$

Let us fix $0<T<\infty$. Then, for any $t \in[0, T]$,

$$
\left\|\breve{\mathbf{E}}_{1}(t)\right\|_{\mathbf{X}} \leq e^{\omega T}\left(\|\breve{\mathbf{E}}(0, \cdot)\|_{\mathbf{X}}+\int_{0}^{t}\|\breve{\mathbf{F}}(s)\|_{\mathbf{X}} d s\right) .
$$

In what follows, constants $c_{i}$ depend only on the coefficients of the problem and $T$ but not on the initial data. By (5.6), Lemma 2.1 and assumptions on $\mathcal{M}$ we have

$$
\left\|\epsilon\left[\stackrel{\circ}{n} \gamma_{W}\right](a)\right\|_{\mathbf{X}} \leq c_{1} \epsilon\|\stackrel{\circ}{n}\|_{L_{1}\left(\mathbb{R}_{+}\right)} .
$$

Similarly, due to the assumptions on $B$ and (2.4), for some $0<\sigma<-s_{C}$

$$
\begin{aligned}
& \left\|\exp \left(\frac{a}{\epsilon} \mathcal{C}_{W}(0)\right)[\mathcal{B}(\stackrel{\circ}{n} \mathbf{k})-\stackrel{\circ}{n} \mathbf{k}(0)]\right\|_{\mathbf{X}} \\
& \leq c_{2}\|\stackrel{\circ}{n}\|_{W_{1}^{1}\left(\mathbb{R}_{+}\right)} \int_{0}^{\infty} e^{-\sigma s / \epsilon} d s \leq \epsilon c_{2} \sigma^{-1}\|\stackrel{\circ}{n}\|_{W_{1}^{1}\left(\mathbb{R}_{+}\right)} .
\end{aligned}
$$

Next, let us consider $\mathbf{F}_{1}(t / \epsilon, a)$. First, we observe that the term $\widetilde{\mathbf{w}}_{0, a}(t / \epsilon, a)$ is well defined due to the assumption that $\stackrel{\circ}{n} \in W_{1}^{1}\left(\mathbb{R}_{+}\right)$, Lemma 2.1 (as $\stackrel{\circ}{\mathbf{w}}=\stackrel{\circ}{\mathbf{n}}-\mathbf{k}(\mathbf{1} \cdot \stackrel{\circ}{\mathbf{n}})$ ) and differentiability of $\mathcal{C}$. Thus, the error estimates involving $\mathbf{F}_{1}$ are all of the form $\int_{0}^{t} e^{-\sigma \frac{t}{\epsilon}} d t \leq \epsilon / \sigma$, where $\sigma$ is as above. Hence

$$
\left\|\int_{0}^{t} \mathcal{G}_{\epsilon}(t-s) \mathbf{F}_{1}(s) d s\right\|_{\mathbf{X}} \leq \epsilon c_{3}\|\stackrel{\circ}{\mathbf{n}}\|_{W_{1}^{1}\left(\mathbb{R}_{+}, \mathbb{R}^{N}\right)}
$$

Let us consider the contribution of $\mathbf{F}_{2}$ to the error. By (3.9) (or (5.7)) we immediately find

$$
\left\|\int_{0}^{t} \mathcal{G}_{\epsilon}(t-s) \mathbf{F}_{2}(s) d s\right\|_{\mathbf{X}} \leq \epsilon T c_{4}\|\stackrel{\circ}{\mathbf{n}}\| \mathbf{X} .
$$

Estimates related to $\mathbf{F}_{3}$ and some other terms of the error are more involved. Before we go on, we mention some additional properties of the operator in (4.17). First, as in [12, Theorem 4.3] $\left(I-\mathcal{B} \mathcal{V}_{\mathcal{K}}\right)^{-1}$ can be extended to a continuous operator on $L_{1}\left([0, T], \mathbb{R}^{N}\right)$ with

$$
\left\|\left(I-\mathcal{B} \mathcal{V}_{\mathcal{K}}\right)^{-1} \mathbf{g}\right\|_{L_{1}\left([0, T], \mathbb{R}^{N}\right)} \leq e^{m T}\|\mathbf{g}\|_{L_{1}\left([0, T], \mathbb{R}^{N}\right)} .
$$

Next, we need estimates of the derivatives of $\bar{n}(\cdot, 0)$. The fact that $\bar{n}(\cdot, 0) \in W_{1, \text { loc }}^{1}\left(\mathbb{R}_{+}\right)$ follows from e.g., [12, Theorem 4.1]. Let us denote $\psi(t)=\bar{n}(t, 0) ; \psi$ is determined from the equation

$$
\psi(t)=\int_{0}^{t} \beta^{*}(a) e^{-\int_{0}^{a} \mu(s) d s} \psi(t-a) d a+\int_{t}^{\infty} \beta^{*}(a) e^{-\int_{a-t}^{a} \mu(s) d s} \stackrel{\circ}{n}(a-t) d a .
$$


If $\psi$ is differentiable then, using the results on differentiability of convolutions (e.g. [1, Proposition 1.3.6] where the assumptions can be relaxed due to the fact that we work with real functions), we get

$$
\psi^{\prime}(t)=\int_{0}^{t} \beta^{*}(a) e^{-\int_{0}^{a} \mu(s) d s} \psi^{\prime}(t-a) d a+g(t),
$$

where

$$
\begin{aligned}
g(t)= & \beta^{*}(t) \psi(0)-e^{-\int_{0}^{t} \mu(s) d s} \beta^{*}(t) \stackrel{\circ}{n}(0) \\
& -\int_{t}^{\infty} \beta^{*}(a) \mu(a-t) e^{-\int_{a-t}^{a} \mu(s) d s} \stackrel{\circ}{n}(a-t) d a-\int_{t}^{\infty} \beta^{*}(a) e^{-\int_{a-t}^{a} \mu(s) d s} \stackrel{\circ}{n^{\prime}}(a-t) d a .
\end{aligned}
$$

By (4.17), ess $\sup _{t \in[0, T]}\left|\psi^{\prime}(t)\right| \leq C$ ess $\sup _{t \in[0, T]}|g(t)|$ and thus, by (5.24),

$$
\begin{aligned}
& |\psi(0)| \leq \operatorname{ess}_{\sup } \sin _{t \in, T]}|\psi(t)| \leq C_{1} \operatorname{ess}_{\sup } \operatorname{se[0,T]}_{t}^{\infty} \beta^{*}(a) e^{-\int_{a-t}^{a} \mu(s) d s}|\stackrel{\circ}{n}(a-t)| d a \\
& \leq C_{2}\|\stackrel{\circ}{n}\|_{L_{1}\left(\mathbb{R}_{+}\right)} \text {. }
\end{aligned}
$$

Similarly

$$
\begin{aligned}
\operatorname{ess} \sup _{t \in[0, T]}\left|\psi^{\prime}(t)\right| & \leq C_{3}\left(\|\stackrel{\circ}{n}\|_{L_{1}\left(\mathbb{R}_{+}\right)}+|\stackrel{\circ}{n}(0)|+\|\stackrel{\circ}{n}\|_{L_{1}\left(\mathbb{R}_{+}\right)}+\|\stackrel{\circ}{n}\|_{L_{1}\left(\mathbb{R}_{+}\right)}\right) \\
& \leq C_{4}\|\stackrel{\circ}{n}\|_{W_{1}^{1}\left(\mathbb{R}_{+}\right)}
\end{aligned}
$$

To estimate $\mathbf{F}_{3}$, first we consider

$$
\begin{aligned}
\frac{1}{\epsilon} \int_{0}^{t} \| & \left(\mathcal{C}_{W}(\cdot)-\mathcal{C}_{W}(0)\right) \widehat{\mathbf{w}}_{0}\left(s, \frac{\cdot}{\epsilon}\right) \|_{\mathbf{X}} d s \\
& =\frac{1}{\epsilon} \int_{0}^{t}\left(\int_{0}^{\infty}\left\|\left(\mathcal{C}_{W}(a)-\mathcal{C}_{W}(0)\right) e^{\frac{a}{\epsilon} \mathcal{C}_{W}(0)} \widehat{\mathbf{w}}_{0}(s, 0)\right\|_{\mathbb{R}^{N}} d a\right) d s \\
& \leq c_{4}^{\prime} \int_{0}^{t}\left(\int_{0}^{\infty} \frac{a}{\epsilon} e^{-\sigma \frac{a}{\epsilon}} d a\right)\left\|\widehat{\mathbf{w}}_{0}(s, 0)\right\|_{\mathbb{R}^{N}} d s=c_{4}^{\prime \prime} \epsilon \int_{0}^{t}\left\|\widehat{\mathbf{w}}_{0}(s, 0)\right\|_{\mathbb{R}^{N}} d s \\
& =c_{4}^{\prime \prime} \epsilon \int_{0}^{t}\|\mathcal{B} \overline{\mathbf{v}}(s, \cdot)-\overline{\mathbf{v}}(s, 0)\|_{\mathbb{R}^{N}} d s \\
& \leq c_{4}^{\prime \prime \prime} \epsilon\left(\int_{0}^{t}\|\bar{n}(s, \cdot)\|_{L_{1}\left(\mathbb{R}_{+}\right)} d s+\int_{0}^{t} \bar{n}(s, 0) d s\right) \leq \epsilon c_{4}^{i v}\|\stackrel{\circ}{n}\|_{L_{1}\left(\mathbb{R}_{+}\right)},
\end{aligned}
$$

where in the last inequality we used (5.23). The next term which requires some reflection is $\widehat{\mathbf{w}}_{0, t}\left(t, \frac{a}{\epsilon}\right)$. First, differentiability of $t \rightarrow \int_{0}^{\infty} B(a) \overline{\mathbf{v}}(t, a) d a=$ : $\int_{0}^{\infty} \bar{n}(t, a) B_{\mathbf{k}}(a) d a$ follows from writing

$$
\begin{aligned}
\int_{0}^{\infty} B_{\mathbf{k}}(a) \bar{n}(t, a) d a= & \int_{0}^{t} B_{\mathbf{k}}(a) e^{-\int_{0}^{a} \mu(s) d s} \bar{n}(t-a, 0) d a \\
& +\int_{t}^{\infty} B_{\mathbf{k}}(a) e^{-\int_{a-t}^{a} \mu(s) d s} \stackrel{\circ}{n}(a-t) d a
\end{aligned}
$$


obtained as in (4.13). Thus, arguing as in the derivation of (5.25),

$$
\begin{aligned}
\frac{d}{d t} & \int_{0}^{\infty} B_{\mathbf{k}}(a) \bar{n}(t, a) d a \\
= & \int_{0}^{t} B_{\mathbf{k}}(a) e^{-\int_{0}^{a} \mu(s) d s} \bar{n}^{\prime}(t-a, 0) d a+B_{\mathbf{k}}(t) e^{-\int_{0}^{t} \mu(s)} \bar{n}(0,0) \\
& -\int_{t}^{\infty} B_{\mathbf{k}}(a) \mu(a-t) e^{-\int_{a-t}^{a} \mu(s) d s} \stackrel{\circ}{n}(a-t) d a \\
& -\int_{t}^{\infty} B_{\mathbf{k}}(a) e^{-\int_{a-t}^{a} \mu(s) d s} \stackrel{\circ}{n^{\prime}}(a-t) d a-e^{-\int_{0}^{t} \mu(s) d s} B_{\mathbf{k}}(t) \stackrel{\circ}{n}(0),
\end{aligned}
$$

for almost all $t>0$, where the values at 0 are well defined by $\stackrel{\circ}{n}$ (and thus $\bar{n}(\cdot, 0)$ ) being $W_{1}^{1}$ functions. Hence

$$
\left\|\widehat{\mathbf{w}}_{0, t}\left(t, \frac{a}{\epsilon}\right)\right\|_{\mathbf{X}} \leq c_{5}^{\prime}\|\stackrel{\circ}{n}\|_{W_{1}^{1}\left(\mathbb{R}_{+}\right)} \int_{0}^{\infty} e^{-\sigma \frac{s}{\epsilon}} d s \leq \epsilon c_{5}^{\prime \prime}\|\stackrel{\circ}{n}\|_{W_{1}^{1}\left(\mathbb{R}_{+}\right)} .
$$

The other two terms in $\mathbf{F}_{3}$ can be easily estimated by $c \epsilon\|\stackrel{\circ}{n}\|_{L_{1}\left(\mathbb{R}_{+}\right)}$(recall that for the continuity of $\bar{n}(t, 0)$ it is enough that $\stackrel{\circ}{n}$ be integrable). Consequently,

$$
\left\|\int_{0}^{t} \mathcal{G}_{\epsilon}(t-s) \mathbf{F}_{3}(s) d s\right\|_{\mathbf{X}} \leq \epsilon c_{5}(T)\|\stackrel{\circ}{n}\|_{W_{1}^{1}\left(\mathbb{R}_{+}\right)} .
$$

Next let us move to $\mathbf{F}_{4}$. Using (5.8) and (5.11), we find

$$
\begin{aligned}
\left\|\int_{0}^{t} \mathcal{G}_{\epsilon}(t-s) \mathbf{F}_{4}\left(\frac{s}{\epsilon}, \cdot\right) d s\right\|_{\mathbf{X}} & \leq e^{\omega T} \int_{0}^{t}\left(\int_{0}^{s}\left\|\mathbf{F}_{4}\left(\frac{s}{\epsilon}, \frac{a}{\epsilon}\right)\right\|_{\mathbb{R}^{N}} d a\right) d s \\
& =\epsilon e^{\omega T} \int_{0}^{t}\left(\int_{0}^{s / \epsilon}\left\|\mathbf{F}_{4}\left(\frac{s}{\epsilon}, \alpha\right)\right\|_{\mathbb{R}^{N}} d \alpha\right) d s .
\end{aligned}
$$

By (5.17), estimates of $\breve{\mathbf{F}}_{4}$ involve four type of expressions. First, by (5.8), the terms involving $\breve{n}_{0}$ satisfy

$$
\begin{aligned}
& \int_{0}^{t}\left(\int _ { 0 } ^ { s / \epsilon } \left(\int_{0}^{\infty}\right.\right.\left.\left.\left\|e^{\left(\frac{s}{\epsilon}-\alpha\right) \mathcal{C}_{W}(a)} \stackrel{\circ}{\mathbf{w}}(a)\right\|_{\left.\mathbb{R}^{N}\right) d a}\right) d \alpha\right) d s \\
& \leq \int_{0}^{t}\left(\int_{0}^{s / \epsilon}\left(\int_{0}^{\infty} e^{-\sigma\left(\frac{s}{\epsilon}-\alpha\right)}\|\stackrel{\circ}{\mathbf{w}}(a)\|_{\mathbb{R}^{N}} d a\right) d \alpha\right) d s \\
& \leq \epsilon\|\stackrel{\circ}{\mathbf{w}}\| \mathbf{x} \int_{0}^{t / \epsilon} \int_{0}^{\eta} e^{-\sigma(\eta-\alpha)} d \alpha d \eta \leq \frac{t\|\stackrel{\circ}{\mathbf{w}}\| \mathbf{x}}{\sigma}
\end{aligned}
$$

Second, we have the terms involving $\breve{\mathbf{w}}_{0}$. By (5.10), the first two contain $\widetilde{\mathbf{w}}_{0}(\tau, a)=$ $e^{\tau C_{W}(a)} \stackrel{\circ}{\mathbf{W}}(a)$ and, similarly to the above, they can be estimated as

$$
\int_{0}^{t} \int_{0}^{s / \epsilon}\left\|e^{\alpha \mathcal{C}_{W}(0)}\left(\int_{0}^{\infty} e^{\left(\frac{s}{\epsilon}-\alpha\right) \mathcal{C}_{W}(a)} \stackrel{\circ}{\mathbf{w}}(a) d a\right)\right\|_{\mathbb{R}^{N}} d \alpha d s
$$




$$
\begin{aligned}
& \leq \int_{0}^{t} \int_{0}^{s / \epsilon} e^{-\sigma \alpha} \int_{0}^{\infty} e^{-\sigma\left(\frac{s}{\epsilon}-\alpha\right)}\|\stackrel{\circ}{\mathbf{w}}(a)\|_{\mathbb{R}^{N}} d a d \alpha d s \\
& \leq \epsilon\|\stackrel{\circ}{\mathbf{w}}\| \mathbf{x} \int_{0}^{t / \epsilon} e^{-\sigma \eta} \eta d \eta \leq \frac{\epsilon\|\stackrel{\circ}{\mathbf{w}}\| \mathbf{x}}{\sigma^{2}} .
\end{aligned}
$$

The last term in (5.10) is $\widetilde{\mathbf{w}}_{0}(\tau, 0)=e^{\tau \mathcal{C}_{W}(0)} \stackrel{\circ}{\mathbf{w}}(0)$ which is well defined under the assumption $\stackrel{\circ}{\mathbf{w}} \in W_{1}^{1}\left(\mathbb{R}_{+}, \mathbb{R}^{N}\right)$. Estimates related to this case are as follows

$$
\begin{aligned}
& \int_{0}^{t} \int_{0}^{s / \epsilon}\left\|e^{\alpha \mathcal{C}_{W}(0)} e^{\left(\frac{s}{\epsilon}-\alpha\right) \mathcal{C}_{W}(0)} \stackrel{\circ}{\mathbf{w}}(0)\right\|_{\mathbb{R}^{N}} d \alpha d s \\
& \leq \int_{0}^{t} \int_{0}^{s / \epsilon} e^{-\sigma \alpha} e^{-\sigma\left(\frac{s}{\epsilon}-\alpha\right)}\|\stackrel{\circ}{\mathbf{w}}(0)\|_{\mathbb{R}^{N}} d \alpha d s \\
& \leq \frac{\epsilon\|\stackrel{\circ}{\mathbf{w}}\|_{\mathbf{W}_{1}^{1}\left(\mathbb{R}_{+}, \mathbb{R}^{N}\right)}}{\sigma^{2}} .
\end{aligned}
$$

The last term requiring our attention is $\epsilon^{-1}\left(\mathcal{C}_{W}(a)-\mathcal{C}_{W}(0)\right) \breve{\mathbf{w}}_{0}\left(\frac{t}{\epsilon}, \frac{a}{\epsilon}\right)$. As above we have two cases depending on the terms in (5.10). The first two can be estimated by the following terms

$$
\begin{aligned}
& \frac{1}{\epsilon} \int_{0}^{t}\left(\int_{0}^{s / \epsilon}\left\|\left(\mathcal{C}_{W}(a)-\mathcal{C}_{W}(0)\right) e^{\alpha \mathcal{C}_{W}(0)} \int_{0}^{\infty} e^{\left(\frac{s}{\epsilon}-\alpha\right) \mathcal{C}_{W}(a)} \stackrel{\circ}{\mathbf{w}}(a) d a\right\|_{\mathbb{R}^{N}} d \alpha\right) d s \\
& \quad \leq c^{\prime} \int_{0}^{t / \epsilon}\left(\int_{0}^{\eta} \alpha e^{-\sigma \alpha} \int_{0}^{\infty} e^{-\sigma(\eta-\alpha)}\|\stackrel{\circ}{\mathbf{w}}(a)\|_{\mathbb{R}^{N}} d a d \alpha\right) d \eta \\
& \quad \leq \frac{1}{2} c^{\prime}\|\stackrel{\circ}{\mathbf{w}}\| \mathbf{x} \int_{0}^{\infty} \eta^{2} e^{-\sigma \eta} d \eta=c^{\prime}\|\stackrel{\circ}{\mathbf{w}}\| \mathbf{x} .
\end{aligned}
$$

The estimate of the term containing $\widetilde{\mathbf{w}}_{0}(\tau, 0)=e^{\tau \mathcal{C}_{W}(0)} \stackrel{\circ}{\mathbf{w}}(0)$ follows similarly:

$$
\frac{1}{\epsilon} \int_{0}^{t}\left(\int_{0}^{s / \epsilon}\left\|\left(\mathcal{C}_{W}(a)-\mathcal{C}_{W}(0)\right) e^{\frac{s}{\epsilon} \mathcal{C}_{W}(0)} \stackrel{\circ}{\mathbf{w}}(0)\right\|_{\mathbb{R}^{N}} d \alpha\right) d s \leq c^{\prime}\|\stackrel{\circ}{\mathbf{w}}\|_{W_{1}^{1}\left(\mathbb{R}_{+}\right)} .
$$

Inserting the above estimates into (5.30) we obtain

$$
\left\|\int_{0}^{t} \mathcal{G}_{\epsilon}(t-s) \mathbf{F}_{4}(s / \epsilon, \cdot) d s\right\|_{\mathbf{X}} \leq \epsilon c_{6}(T)\|\stackrel{\circ}{\mathbf{n}}\|_{W_{1}^{1}\left(\mathbb{R}_{+}, \mathbb{R}^{N}\right)} .
$$

It remains to estimate the contribution of the boundary terms. For this we use Eq. (4.14) in which $\mathcal{K}(a)=-\mathcal{M}(a)+\epsilon^{-1} \mathcal{C}(a)$ and, by (4.12),

$$
\left\|\breve{\mathbf{E}}_{2}(t)\right\|_{\mathbf{X}} \leq\|\boldsymbol{\omega}(t, \cdot)\| \mathbf{X}=\int_{0}^{t}\left\|\left[\left(I-\mathcal{B} \mathcal{V}_{\mathcal{K}}\right)^{-1} \mathbf{g}\right](t-a)\right\|_{\mathbb{R}^{N}} d a .
$$

Therefore, by (5.23), $\left\|\breve{\mathbf{E}}_{2}(t)\right\|_{\mathbf{X}} \leq\|\boldsymbol{\omega}(t, \cdot)\|_{\mathbf{X}} \leq c_{7}(T)\|\mathbf{g}\|_{L_{1}\left([0, T], \mathbb{R}^{N}\right)}$. Since $t \rightarrow \bar{n}(t, \cdot)$ is a mild solution to (3.10), it is strongly continuous and thus the $L_{1}([0, T])$ norms of the terms $\epsilon \int_{0}^{\infty} \mathbf{1} \cdot B(s) \overline{\mathbf{w}}_{1}(t, s) d s, \epsilon \mathcal{B}_{W} \overline{\mathbf{w}}_{1}(t, \cdot)$ and $\epsilon \boldsymbol{\gamma}_{W}(0) \int_{0}^{\infty} \bar{n}(t, s) \beta^{*}(s) d s$ are bounded by $\epsilon c_{8}(T)\|\stackrel{\circ}{n}\| L_{1}\left(\mathbb{R}_{+}\right)$, where $c_{8}(T)$ is related to the type of the solution $\bar{n}$. Next we consider the corner layer terms: 
$\int_{0}^{\infty} \breve{n}_{0, \epsilon}(t, s) \beta^{*}(s) d s, \quad \int_{0}^{\infty} \mathbf{1} \cdot B(s) \breve{\mathbf{w}}_{0, \epsilon}(t, s) d s, \quad\left[\mathcal{B}\left(\breve{n}_{0, \epsilon}(t, \cdot) \mathbf{k}(\cdot)\right)\right], \quad\left[\mathcal{B} \breve{\mathbf{w}}_{0, \epsilon}(t, \cdot)\right]$,

and, using (3.4), (5.8), (5.11) and (5.10), we see that all terms in these expressions have the generic form

$$
\int_{0}^{t} \mathbf{B}_{1}(s)\left(\int_{0}^{\infty} \mathbf{B}_{2}(\sigma) e^{\frac{t-s}{\epsilon} \mathcal{C}_{W}(\sigma)} \mathbf{x}(\sigma) d \sigma\right) d s \quad \text { or } \quad \int_{0}^{t} \mathbf{B}_{1}(s) e^{\frac{t-s}{\epsilon} \mathcal{C}_{W}(0)} \mathbf{x}(0) d s,
$$

where $\mathbf{x} \in W_{1}^{1}\left(\mathbb{R}_{+}, \mathbb{R}^{k}\right), \mathbf{B}_{i} \in L_{\infty}\left(\mathbb{R}_{+}, \mathcal{L}\left(\mathbb{R}^{k}, \mathbb{R}^{l}\right)\right), i=1,2$, with $k, l$ equal to either 1 or $n$. Hence, the estimates of the $L_{1}([0, T])$ norm of them are of the same type as (5.30) combined with (5.31)-(5.33). Finally, the estimates of the boundary layer terms $\int_{0}^{\infty} \mathbf{1} \cdot B(s) \widehat{\mathbf{w}}_{0, \epsilon}(t, s) d s$ and $\mathcal{B} \widehat{\mathbf{w}}_{0, \epsilon}(t, \cdot)$ follow from $(5.28)$ due to the boundedness of the coefficients of $B$.

Summarizing, we have proved that for any $T<\infty$ there is a constant $C=$ $C(T, \mathcal{M}, B, \mathcal{C})$ such that

$$
\begin{aligned}
& \| \mathbf{n}_{\epsilon}(t, a)-\left(\overline{\mathbf{v}}(t, a)+\breve{\mathbf{v}}\left(\frac{t}{\epsilon}, \frac{a}{\epsilon}\right), \epsilon \overline{\mathbf{w}}_{1}(t, a)\right. \\
& \left.\quad+\tilde{\mathbf{w}}_{0}\left(\frac{t}{\epsilon}, a\right)+\hat{\mathbf{w}}_{0}\left(t, \frac{a}{\epsilon}\right)+\breve{\mathbf{w}}_{0}\left(\frac{t}{\epsilon}, \frac{a}{\epsilon}\right)\right) \|_{\mathbf{X}} \\
& \leq \epsilon C\|\stackrel{\circ}{\mathbf{n}}\|_{W_{1}^{1}\left(\mathbb{R}_{+}, \mathbb{R}^{N}\right)}
\end{aligned}
$$

uniformly in $t \in[0, T]$. However, similarly to (5.31), we find

$$
\begin{aligned}
\left\|\breve{n}_{0}\left(\frac{t}{\epsilon}, \cdot\right)\right\|_{L_{1}\left(\mathbb{R}_{+}\right)} & \leq c_{9} \int_{0}^{t}\left(\int_{0}^{\infty} e^{-\sigma\left(\frac{t-a}{\epsilon}\right)}\|\stackrel{\circ}{\mathbf{w}}(r)\|_{\mathbb{R}^{N}} d r\right) d a \\
& \leq \epsilon c_{9}\|\stackrel{\circ}{\mathbf{w}}\|_{\mathbf{X}} e^{-\sigma \frac{t}{\epsilon}} \int_{0}^{t / \epsilon} e^{\sigma \frac{a}{\epsilon}} d a \leq \frac{\epsilon c_{9}\|\stackrel{\circ}{\mathbf{w}}\| \mathbf{X}}{\sigma}
\end{aligned}
$$

and, as in (5.32) and (5.33),

$$
\begin{aligned}
\left\|\breve{\mathbf{w}}_{0}\left(\frac{t}{\epsilon}, \cdot\right)\right\|_{\mathbf{X}} & \leq c_{10}\|\stackrel{\circ}{\mathbf{w}}\|_{W_{1}^{1}\left(\mathbb{R}_{+}, \mathbb{R}^{N}\right)} \int_{0}^{t} e^{-\sigma \frac{a}{\epsilon}} d a \\
& \leq \epsilon c_{10}\|\stackrel{\circ}{\mathbf{w}}\|_{W_{1}^{1}\left(\mathbb{R}_{+}, \mathbb{R}^{N}\right)}\left(\max _{z \in \mathbb{R}_{+}} z e^{-\sigma z}\right) \leq \frac{\epsilon c_{10}}{\sigma e}\|\stackrel{\circ}{\mathbf{w}}\|_{W_{1}^{1}\left(\mathbb{R}_{+}, \mathbb{R}^{N}\right)} .
\end{aligned}
$$

By (3.10) we find, as in (5.22) without integration with respect to $s$, that

$$
\left\|\epsilon \overline{\mathbf{w}}_{1}(t, \cdot)\right\| \mathbf{X} \leq \epsilon c_{11}(T)\|\stackrel{\circ}{\mathbf{n}}\|_{\mathbf{X}} .
$$

Finally, (5.28) gives an $O(\epsilon)$ estimate of $\widehat{\mathbf{w}}_{0}$. Combining the above estimates, we can move $\epsilon \overline{\mathbf{w}}_{1}$ as well as the boundary and corner layer terms to the right-hand side and re-write (5.35) as

$$
\left\|\mathbf{n}_{\epsilon}(t, a)-\left(\overline{\mathbf{v}}(t, a), \tilde{\mathbf{w}}_{0}\left(\frac{t}{\epsilon}, a\right)\right)\right\|_{L_{1}\left(\mathbb{R}_{+}, \mathbb{R}^{N}\right)} \leq \epsilon C(T, \mathcal{M}, B, \mathcal{C})\|\stackrel{\circ}{\mathbf{n}}\|_{W_{1}^{1}\left(\mathbb{R}_{+}, \mathbb{R}^{N}\right)}
$$

uniformly in $t \in[0, T]$ which, written in components, gives (1.8). 
COROLLARY 5.2. Let us assume that $\mathcal{C}, B$ and $\mathcal{M}$ satisfy assumptions introduced in Sect. 2.1 and $\mathbf{n}_{\epsilon}(t, a):=\left[\mathcal{G}_{\epsilon}(t) \stackrel{\circ}{\mathbf{n}}\right](a)=n_{\epsilon}(t, a) \mathbf{k}(a)+\mathbf{w}_{\epsilon}(t, a)$ be a solution to (1.1). Then, for any $\stackrel{\circ}{\mathbf{n}} \in L_{1}\left(\mathbb{R}_{+}, \mathbb{R}^{N}\right)$, we have

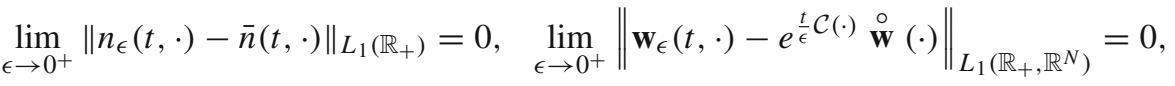
uniformly on $[0, T]$.

Proof. This corollary follows from density of $W_{1}^{1}\left(\mathbb{R}_{+}, \mathbb{R}^{N}\right)$ in $L_{1}\left(\mathbb{R}_{+}, \mathbb{R}^{N}\right)$ and equiboundedness of the converging families of operators with respect to $\epsilon$, see e.g. [6].

\section{Numerical illustration}

\subsection{Numerical algorithm}

To provide a numerical illustration of the asymptotic expansion developed in Sect. 3 we follow [7]. First we give numerical approximations to $\mathbf{n}, \bar{n}, \tilde{\mathbf{n}}, \hat{\mathbf{n}}$ and $\breve{\mathbf{n}}$. We begin with $\mathbf{n}$. Let $\mathcal{K}=-\mathcal{M}+\frac{1}{\epsilon} \mathcal{C}$ and $\mathcal{V}_{\mathcal{K}, a_{0}}(a)$ be the fundamental solution matrix to the equation $\mathcal{V}^{\prime}(a)=\mathcal{K}(a) \mathcal{V}(a), V\left(a_{0}\right)=I$, then Lemma 4.2 implies that

$$
\mathbf{n}(t, 0)=\int_{0}^{t} B(a) \mathcal{V}_{\mathcal{K}, 0}(a) \mathbf{n}(t-a, 0) d t+\int_{0}^{\infty} B(a+t) \mathcal{V}_{\mathcal{K}, a}(a+t) \stackrel{\circ}{\mathbf{n}}(a) d a
$$

and

$$
\mathbf{n}(t, 0)= \begin{cases}\mathcal{V}_{\mathcal{K}, a-t}(a) \stackrel{\circ}{\mathbf{n}}(a-t), & a>t, \\ \mathcal{V}_{\mathcal{K}, 0}(a) \mathbf{n}(t-a, 0), & a<t .\end{cases}
$$

Formulae (6.1), (6.2) suggest the following algorithm: first, we solve the Volterra integral equation (6.1) for $\mathbf{n}(t, 0)$; second, we recover $\mathbf{n}(t, a)$ by integrating linear ODEs along the characteristic lines using (6.2).

To solve (6.1) in $[0, T]$ we set $\mathbf{F}(t)=\int_{0}^{\infty} B(a+t) \mathcal{V}_{\mathcal{K}, a}(a+t) \stackrel{\circ}{\mathbf{n}}(a) d a$, introduce a grid $\left\{t_{k}\right\}_{1 \leq k \leq M}$ and apply $A(\alpha)$ stable, 4 -step, order 4 BDF formula to

$$
\mathbf{u}(t, s)=\mathbf{F}(t)+\int_{0}^{s} B(t-a) \mathcal{V}_{\mathcal{K}, 0}(t-a) \mathbf{n}(a, 0) d a, \quad \mathbf{n}(t, 0)=\mathbf{u}(t, t) .
$$

This yields the algorithm

$$
\begin{aligned}
\sum_{j=0}^{4} a_{j} \mathbf{u}\left(\cdot, t_{k-j}\right) & =\tau_{k-1} B\left(\cdot-t_{k-j}\right) \mathbf{v}\left(\cdot-t_{k-j}, t_{k-j}\right), \quad \mathbf{n}\left(t_{k}, 0\right)=\mathbf{u}\left(t_{k}, t_{k}\right), \\
\mathbf{u}\left(\cdot, t_{k}\right) & =\mathbf{F}(\cdot)+\tau_{0} \sum_{j=-4}^{-1} w_{0, j} B\left(\cdot-t_{j}\right) \mathbf{v}\left(\cdot, t_{j}\right), \quad-4 \leq k \leq 0, \\
\mathbf{v}_{s}\left(s, t_{k}\right) & =\mathcal{K}(s) \mathbf{v}\left(s, t_{k}\right), \quad \mathbf{v}\left(0, t_{k}\right)=\mathbf{n}\left(t_{k}, 0\right),
\end{aligned}
$$


where $a_{j}$ are the coefficients of the BDF formula, $w_{0, j}$ are the coefficients of a starting procedure and $\tau_{k}=t_{k}-t_{k-1}$.

The method (6.3)-(6.5) requires one evaluation of $\mathbf{F}(t)$ per integration step. In our implementation this is done by means of the three-points, composite Gauss-Lobatto quadrature. To accomplish numerical integration of linear ODEs in (6.5) the algebraically stable, stiffly accurate, 3-stage RadauIIa Runge-Kutta method of order 5 is employed. It can be shown that under the assumptions on $\mathcal{C}, \mathcal{B}$ and $\mathcal{M}$ the algorithm (6.3)-(6.5) converges with order four to $\mathbf{n}(t, 0)$ (i.e. the global error is $O\left(\max _{k} \tau_{k}^{4}\right)$ ) in any finite interval $[0, T]$, moreover the convergence is uniform for all $\epsilon>0$.

Consider now the bulk approximation $\bar{n}$. It satisfies the scalar Eq. (1.5), (1.6) which is of the same form as the original model (1.1)-(1.3). For this reason, the numerical approximation to $\bar{n}$ is obtained in the same way as for $\mathbf{n}$.

The initial and the boundary layer corrections involve solution of linear ODEs. Numerical approximations in these cases are trivial, moreover, the corrections are needed only in $O(\epsilon)$ neighborhood of the boundaries $a=0$ and $t=0$.

Finally, the corner layer equation is of the same form as the original system, thus, the technique (6.3)-(6.5) is applicable. Once again, $\breve{\mathbf{n}}$ vanishes outside $O(\epsilon)$ neighborhood of the corner point and only local approximation is required.

\subsection{Computational example}

For numerical simulaions we take a simple two dimensional problem with $\mathcal{M}=$ $\operatorname{diag}\{1,1\}, B=\operatorname{diag}\{1,2\}$ and $\mathcal{C}=\left\{c_{i j}\right\}_{1 \leq i, j \leq 2}$, where $c_{11}=c_{22}=-1$ and $c_{12}=c_{21}=1$. As the initial condition we take $\mathbf{n}(a)=\left(e^{-a}, e^{-2 a}\right)$. We take the perturbation parameter $\epsilon=10^{-3}$. We note that $\stackrel{\circ}{\mathbf{n}} \in D(\mathcal{A})$ and thus the solution to the full problem is continuous. In this problem $\mathbf{k}=(1 / 2,1 / 2)$ and the aggregated equation is given by

$$
n_{t}+n_{a}=-n, \quad \stackrel{\circ}{n}(a)=e^{-a}+e^{-2 a}, \quad n(t, 0)=\frac{3}{2} \int_{0}^{\infty} n(t, a) d a .
$$

It is clear that $\stackrel{\circ}{n}$ does not satisfy the compatibility condition and the solution to the aggregated problem only exists in the mild sense as it is discontinuous along the characteristic line $a=t$ (see right diagram of Fig. 1).

Figures 1 and 2 provide illustration to the asymptotic theory developed in Sects. 3-5. The right diagram of Fig. 1 shows the bulk approximation $\bar{n} k_{1}$ to the first component of the solution $\mathbf{n}=\left(n_{1}, n_{2}\right)$; that is $n_{1}$. Its error $\bar{e}_{1}$ is given in the upper left diagram of Fig. 2. One can clearly see that $\bar{n} k_{1}$ provides a good uniform approximation to the solution $n_{1}$ of the perturbed problem everywhere except near the boundaries and at the characteristic line $a=t$. The upper right diagram of Fig. 2 shows the effect of the initial layer correction. The effects of the boundary and the corner layers corrections are depicted in the lower left and the lower right diagrams of Fig. 2, respectively.

Figure 3 illustrates Theorem 5.1. The left part of the figure plots the bulk approximation error $\|\mathbf{n}(t, \cdot)-\overline{\mathbf{n}}(t, \cdot)\|_{L_{1}\left(\mathbb{R}_{+}, \mathbb{R}^{N}\right)}$ as a function of time. The error is large in 

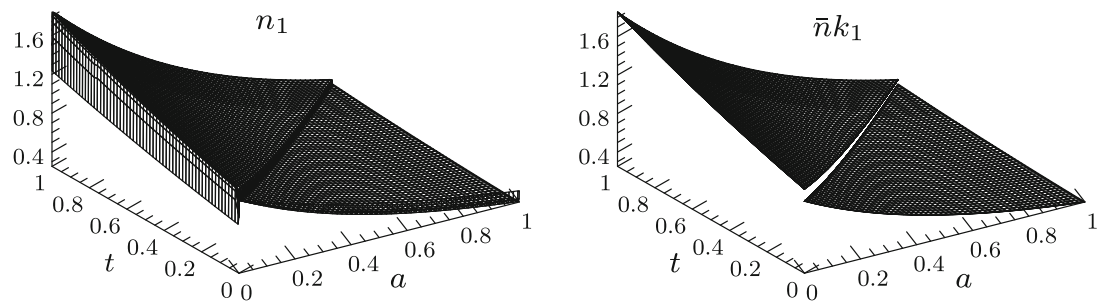

Figure 1. The first component $n_{1}$ of the solution with initial data satisfying the compatibility condition (left) and its bulk approximation $\bar{n} k_{1}$ (right), $\epsilon=10^{-3}$
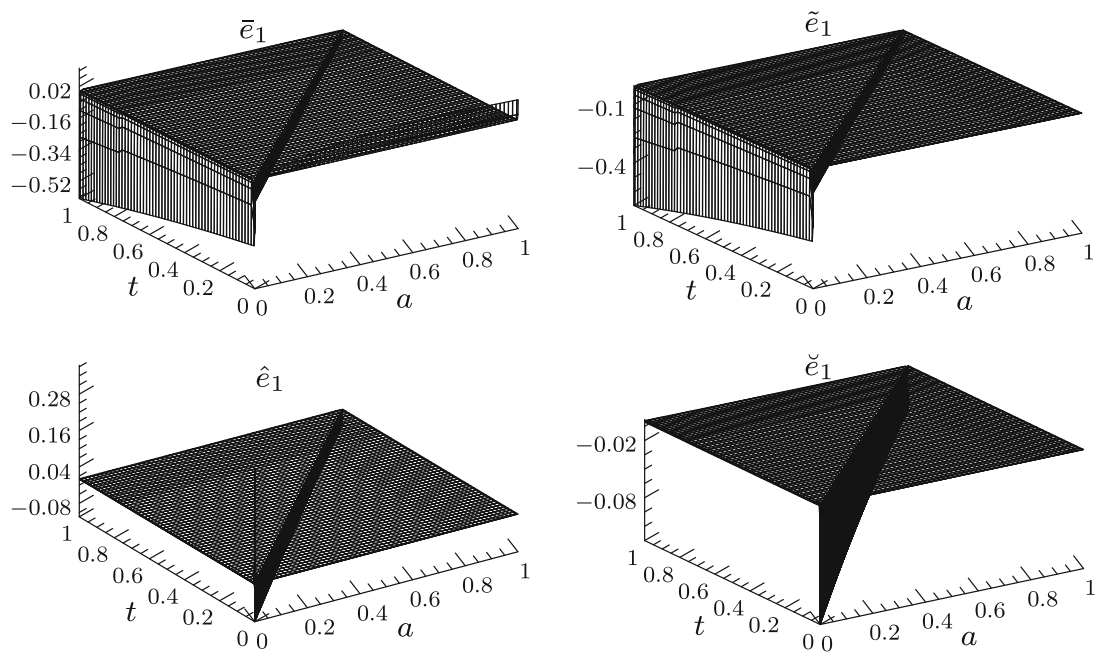

Figure 2. The bulk approximation error and the effect of the initial, boundary and corner layer corrections, $\epsilon=10^{-3}$

a $O(\epsilon)$ neighborhood of $t=0$ and is of magnitude $O(\epsilon)$ away from the origin. The errors obtained after corrections are given in the right diagram. As predicted by Theorem 5.1, the initial layer correction alone reduces the error to $O(\epsilon)$ everywhere in the time interval. Using the boundary and the corner layer corrections slightly improves the error but does not change its order.

\section{Conclusions}

The main aim of this paper is to show how the application of classical techniques of asymptotic analysis and, in particular, of the Chapman-Enskog procedure, can yield the aggregation of variables in a more systematic way and deliver a simpler approximation formula than the ad hoc method of [2]. It may seem strange that the constructed elaborate hierarchy of layers is only used in intermediate steps of the analysis but, 

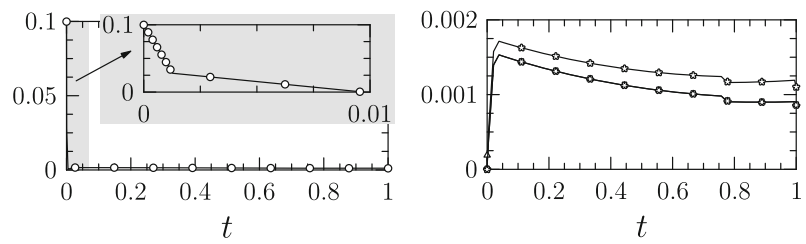

Figure 3. The $L_{1}\left(\mathbb{R}_{+}, \mathbb{R}^{N}\right)$ errors before (left) and after (right) layer corrections: bulk only (circles), bulk and initial layer (5-point stars), bulk, initial and boundary layers (triangles) and bulk, initial, boundary and corner layers (6-point stars), $\epsilon=10^{-3}$

apart from the initial layer, does not appear in the final approximation. In our opinion this is one of the advantages of the method which, while providing all potentially significant terms of the expansion, allows for discarding all these which are not absolutely necessary. In our case the absence of the boundary and the corner layers in the final approximation is due to the choice of the state space $L_{1}\left(\mathbb{R}_{+}, \mathbb{R}^{N}\right)$. The norm of $L_{1}\left(\mathbb{R}_{+}, \mathbb{R}^{N}\right)$ averages the terms of layers which decay exponentially fast in $a / \epsilon$ and thus makes them negligible. Clearly, as can be seen from the numerical experiments, these terms would be essential to get a uniform approximation if the $L_{\infty}\left(\mathbb{R}_{+}, \mathbb{R}^{N}\right)$ norm was used. On the other hand, there are approximation techniques which use an integral norm also with respect to $t$. In such a case the initial layer becomes negligible as well, see e.g. [14]. We also note that the boundary layer becomes important in the diffusion approximation of the stationary transport equation, see e.g. [10, Chap. XXI].

Finally we note that we have considered the simplest model the relevance of which in realistic population theory is limited. Various generalizations are possible. For instance, staying within linear models one can consider reducible transition matrices which aggregation of which results in coupled McKendrick models of lower dimension. On the other hand, age structured epidemiological models offer examples of (1.1) type models in which the coupling is provided by nonlinear ML matrices, see [12]. Such models are subject of current research and we believe that this paper provides a solid foundation for their analysis.

Open Access. This article is distributed under the terms of the Creative Commons Attribution Noncommercial License which permits any noncommercial use, distribution, and reproduction in any medium, provided the original author(s) and source are credited.

\section{REFERENCES}

[1] Arendt, W., BAtty, CH. J. K., Hieber, M., Neubrander, F., Vector-valued Laplace transforms and Cauchy problems, Birkhäuser Verlag, Basel, 2001.

[2] Arino, O., SÁnchez, E., Bravo de la Parra, R. And Auger, P., A singular perturbation in an age-structured population model, SIAM Journal on Applied Mathematics, 60(2), 1999, 408-436.

[3] Auger, P., Bravo de la Parra, R., Poggiale, J.-C., Sánchez, E. and Nguyen-HuU, T., Aggregation of Variables and Application to Population Dynamics, in: Magal, P. and Ruan, S. 
(eds), Structured Population Models in Biology and Epidemiology, LMN 1936, Springer Verlag, Berlin, 2008, 209-263.

[4] Banasiak, J., ARlotti, L., Perturbations of positive semigroups with applications, Springer, London, 2006.

[5] BANASIAK, J., Asymptotic analysis of singularly perturbed dynamical systems, in: A. Abdulle, J. Banasiak, A. Damlamian and M. Sango (eds), Multiscale Problems in Biomathematics, Physics and Mechanics: Modelling, Analysis and Numerics, GAKUTO Internat. Ser. Math. Sci. Appl. 31, Gakkotosho, Tokyo, 2009, 221-255.

[6] BANASIAK, J., BOBROWSKI, A., Interplay between degenerate convergence of semigroups and asymptotic analysis, J. Evol. Equ., 9(2), 2009, 293-314.

[7] BANASIAK, J. AND SHINDIN, S., Chapman-Enskog asymptotic procedure in structured population dynamics, Il Nuovo Cimento C, 33(1), (2010), 31-38.

[8] BoBrowski, A., Functional analysis for probability and stochastic processes, Cambridge University Press, Cambridge, 2005

[9] Bravo de la Parra, R., Arino, O., SÁnchez, E., Auger, P., A model for an age-structured population with two time scales, Math. Comput. Modelling 31(4-5), (2000), 17-26.

[10] Dautrey, R. AND LiOns J.- L., Mathematical Analysis and Numerical Methods for Science and Technology, vol. 6, Springer Verlag, Berlin, 2000.

[11] Engel, K.- J. And NAgel, R., One-Parameter Semigroups for Linear Evolution Equations, Springer Verlag, New York, 2000.

[12] IANNELLI, M., Mathematical theory of age-structured population dynamics, Applied Mathematics Monographs 7, Consiglio Nazionale delle Ricerche (C.N.R.), Giardini, Pisa, 1995.

[13] Kato, T., Perturbation Theory for Linear Operators, 2nd ed., Springer Verlag, Berlin, 1984.

[14] Lions, P. L. And TosCANI, G., Diffusive limit for finite velocity Boltzmann kinetic models, Rev. Mat. Iberoamericana 13(3) (1997), 473-513.

[15] Lisi, M., TOTARO, S., The Chapman-Enskog procedure for an age-structured population model: initial, boundary and corner layer corrections, Math. Biosci., 196(2), 2005, 153-186.

[16] Martin, R. H., Nonlinear Operators \& Differential Equations in Banach Spaces, Wiley, New York, 1976.

[17] Mika, J. R. AND BANASIAK, J., Singularly Perturbed Evolution Equations with Applications in KineticTheory, World Sci., Singapore, 1995.

[18] SEneta, E., Nonnegative matrices and Markov chains, 2nd ed., Springer Series in Statistics. Springer-Verlag, New York, 1981.

[19] WebB, G. F., Theory of Nonlinear Age-dependent Population Dynamics, Marcel Dekker, New York, 1985.

\author{
J. Banasiak, A. Goswami \\ S. Shindin \\ School of Mathematical Sciences, \\ University of KwaZulu-Natal, \\ Durban, South Africa \\ E-mail:banasiak@ukzn.ac.za \\ J. Banasiak \\ Institute of Mathematics, \\ Technical University of Łódź, \\ Łódź, Poland \\ A. Goswami \\ E-mail: goswamia@ukzn.ac.za \\ S. Shindin \\ E-mail: shindins@ukzn.ac.za
}

